\title{
Milk fat globule-EGF factor 8 suppresses the aberrant immune response of systemic lupus erythematosus-derived neutrophils and associated tissue damage
}

\author{
Wei Huang ${ }^{1,2}$, Jiyuan $\mathrm{Wu}^{3}$, Huiqin Yang ${ }^{4}$, Yin Xiong ${ }^{5}$, Rui Jiang ${ }^{2}$, Tianpen Cui ${ }^{\star \star 2,6}$ and Duyun Ye $\mathrm{Y}^{\star, 1,6}$
}

\begin{abstract}
Abnormal features of the systemic lupus erythematosus (SLE)-derived neutrophils, promoted aberrant immune response, have inspired new studies of the induction of autoimmunity and the development of organ damage in SLE. In this study, we explore the effect of milk fat globule-EGF factor 8 (MFG-E8) on the aberrant nitrification features in pristane-induced lupus. SLE patients and mice with pristane-induced lupus develop autoantibodies associated with MFG-E8 overproduction. However, the deletion of MFG-E8 leads to uncontrolled early pulmonary and peritoneal inflammation and tissue damage in mice with pristane-induced lupus. Consistent with these findings, MFG-E8-deficient mice that are exposed to pristane show enhanced neutrophil accumulation and increased neutrophil death, including apoptosis, necrosis and NETosis, as well as impaired phagocytosis of macrophages. The consequences are the expansion of diffuse pulmonary hemorrhage, increased anti-nuclear antibody, antidsDNA antibody and anti-neutrophil cytoplasmic antibody levels, and enhanced immune complexes deposition and neutrophil extracellular traps (NETs) formation in the lung and kidney tissues of MFG-E8-deficient mice exposed to pristane. In patients with SLE and mice with pristane-induced lupus, neutrophil accumulation is elevated, which depends on higher expression of the surface receptor CXCR2. After pretreatment with recombinant MFG-E8, the surface expression of CXCR2 on neutrophil is downregulated, and the MFG-E8 deletion increase CXCR2 expression by $\sim 40 \%$. These studies indicate that MFG-E8 reduces neutrophil migration and NETosis via downregulating surface CXCR2 expression in parallel with its role in the phagocytosis of apoptotic neutrophils, suggesting that MFG-E8 may serve as a therapeutic agent for attenuating the early inflammatory responses of SLE and protect patients from lupus-related damage.
\end{abstract}

Cell Death and Differentiation (2017) 24, 263-275; doi:10.1038/cdd.2016.115; published online 21 October 2016

Systemic lupus erythematosus (SLE) is characterized by the overproduction of autoantibodies with multiple organ damage owing to the loss of tolerance to self-antigens. ${ }^{1}$ Although major effects have been attributed to the study of the dysregulation of adaptive immunity, ${ }^{2}$ in recent years, the active role of innate immune system in driving the autoimmune response and tissue damage in SLE has attracted more attention. ${ }^{3}$ Neutrophils have both a destructive role and a driving role in the pathogenesis of various inflammatory or autoimmune diseases, ${ }^{4}$ and previous studies showed that the phenotype and function of neutrophils derived from SLE patients displayed a number of abnormal features, such as increased infiltration, increased apoptosis and impaired phagocytosis, which may lead to neutropenia, the accumulation of apoptotic cells and the enrichment of low-density granulocytes (LDGs). ${ }^{3,5,6}$ Circulating immune complexes (CICs) composed of nuclear antigens and autoantigens against DNA/ribonucleoprotein, which are likely derived from the death of neutrophils, recently described as NETosis, result in the externalization of a meshwork of chromatin fibers. ${ }^{7-9}$ Neutrophil NETosis has an important role in organ damage and vascular cytotoxicity in lupus patients. ${ }^{10}$

\footnotetext{
${ }^{1}$ Department of Pathophysiology, Tongji Medical College, Huazhong University of Science and Technology, Wuhan, China; ${ }^{2}$ Laboratory of Clinical Immunology, Wuhan No.1 Hospital, Tongji Medical College, Huazhong University of Science and Technology, Wuhan, China; ${ }^{3}$ Department of Dermatology, Wuhan No.1 Hospital, Tongji Medical College, Huazhong University of Science and Technology, Wuhan, China; ${ }^{4}$ Department of Rheumatology and Immunology, Wuhan No.1 Hospital, Tongji Medical College, Huazhong University of Science and Technology, Wuhan, China and ${ }^{5}$ Department of Laboratory Medicine, Hubei University of Chinese Medicine, Wuhan, China ${ }^{*}$ Corresponding author: T Cui, Laboratory of Clinical Immunology, Wuhan No.1 Hospital, Tongji Medical College, Huazhong University of Science and Technology, Wuhan 430022, China. Tel: +86 27 85332628; E-mail: tianpencui @ 126.com

or D Ye, Department of Pathophysiology, Tongji Medical College, Huazhong University of Science and Technology, Wuhan 430030, China. Tel: +86 156 07124302 ; Fax: +86 0278369 2816; E-mail: yedy@mails. tjmu.edu.cn

${ }^{6}$ These authors contributed equally to this work.

Abbreviations: ANA, anti-nuclear antibody; ANCA, anti-neutrophil cytoplasmic antibody; ALI, acute lung injury; BALF, bronchoalveolar lavage fluid; BMDNs, bone marrow-derived neutrophils; CICs, circulating immune complexes; $\mathrm{Cr}$, creatinine; DPH, diffuse pulmonary hemorrhage; GRK2, G protein-coupled receptor kinase 2; HMGB1, high mobility group box-1 protein; ilCs, immobilized immune complexes; LDGs, low-density granulocytes; MFG-E8, milk fat globule-EGF factor 8; MPO-ANCA, myeloperoxidase-ANCA; NADPH, nicotinamide adenine dinucleotide phosphate; NETs, neutrophil extracellular traps; NO, nitric oxide; PAD4, peptidylarginine deiminase 4; PBCs, peripheral blood cells; PBS, phosphate buffered saline; PMA, phorbol 12-myristate 13-acetate; PR3-ANCA, proteinase 3-ANCA; PS, phosphatidylserine; rhMFG-E8, recombinant human MFG-E8; rmMFG-E8, recombinant murine MFG-E8; ROS, reactive oxygen species; SLE, system lupus erythematosus; SOE, sarcosine oxidase enzymatic; WT, wild type

Received 30.4.16; revised 27.8.16; accepted 16.9.16; Edited by S Nagata; published online 21.10.16
} 
Milk fat globule-EGF factor 8 (MFG-E8) is a glycoprotein that interacted with phagocytes to stimulate the uptake of the apoptotic cells, ${ }^{11}$ and ubiquitously expressed various organs and cells. ${ }^{12}$ MFG-E8-deficient follicular dendritic cells and tingible body macrophages in the germinal centers cause impaired clearance of apoptotic cells, and then these apoptotic cells undergo secondary necrosis and release proinflammatory or immunogenic self-antigens such as high mobility group box-1 protein and dsDNA, ultimately leading to autoimmune disease similar to SLE. ${ }^{13-15}$ The effect of MFG-E8 on engulfment is strongly limited by a dose-dependent but bellshaped pattern: MFG-E8 enhances the engulfment of apoptotic cells at lower concentrations, but it inhibits engulfment at higher concentrations. ${ }^{16,17}$ Moreover, MFG-E8 deficiency promotes immune responses to self-antigens not only by delaying the clearance of dying cells but also by altering intracellular processing, leading to enhanced self-antigen presentation. ${ }^{18}$ In addition, as mutations in the $\mathrm{C} 1$ domain of MFG-E8 often impair the binding to PS of apoptotic cells, its polymorphism is related to SLE predisposition. ${ }^{19}$

With its direct regulatory function on neutrophils, MFG-E8 has a potentially therapeutic role in treating acute lung injury. ${ }^{20,21}$ Recently, studies have revealed that the administration of recombinant murine MFG-E8 (rmMFG-E8) reduced neutrophil migration. The mechanism was identified as MFGE8 binding to its receptor $a_{v} \beta_{3}$ integrin transduced downstream MAP kinase (P38 and ERK) signaling, and then the activated MAP kinases upregulated $G$ protein-coupled receptor kinase 2, which negatively regulated the surface levels of CXCR2, a chemokine receptor expressed on the surface of neutrophils. $^{21,22}$ MFG-E8, its effect on neutrophils, which have a central role in the pathogenesis of SLE, is not clearly understood.

\section{Results}

MFG-E8 is essential for SLE patients and mice with pristane-induced lupus. The serum MFG-E8 levels were significantly increased $(>1 \mathrm{ng} / \mathrm{ml})$ in 18 of the $51(35.30 \%)$ active, treated or non-treated SLE patients compared with the healthy controls (a mean concentration of $2.104 \pm 0.600$ versus $0.720 \pm 0.278 \mathrm{ng} / \mathrm{ml} ; P<0.05$; Figure $1 \mathrm{a})$. In the mice with pristane-induced lupus, the serum MFG-E8 level was elevated after 24 weeks, and the MFG-E8 levels in bronchoalveolar lavage fluid (BALF), peritoneal cavity and lung tissues were significantly increased in the early phase at 2 weeks (Figures $1 \mathrm{~b}$ and $\mathrm{c}$ ).

The absence of MFG-E8 enhances early lung inflammation in response to pristine. We successfully constructed MFG-E8 knockout mice in the C57BL/6j background (Supplementary Figure 1). In the early phase, the Mfge $^{-/-}$ mice showed increased total BAL leukocyte counts including neutrophils (polymorphonuclear neutrophils, PMNs) and macrophages at $16 \mathrm{~h}, 5$ days, 10 days and 14 days after the pristane injection (Figure 1d). In particular, the numbers of PMNs and macrophages were significantly increased in the lungs of the $\mathrm{Mfge}^{-/-}$mice at $16 \mathrm{~h}$ and 14 days of pristane treatment. As the i.p. pristane injection frequently resulted in diffuse pulmonary hemorrhage (DPH) within a few weeks in C57BL/6 mice, ${ }^{23,24}$ in this study, the $\mathrm{Mfge8}^{-1-}$ mice developed more complete DPH than WT mice after a 2-week pristane treatment, displaying a higher DPH prevalence and $\mathrm{DPH}$ score and increased protein and neutrophil infiltration (Figure 1e). The organization of the lymphoid nodules (white pulps) in the spleen of the pristane-treated $\mathrm{Mfge8}^{-/-}$mice was disorganized and the number of megakaryocytes was increased compared with the WT mice (Supplementary Figure 2a). Similarly, the livers from the pristane-treated $\mathrm{Mfge}^{-/}$mice showed neutrophil and erythroid lineage infiltration; meanwhile, the kidney showed glomerular myeloid cells infiltration (Supplementary Figures $2 \mathrm{~b}$ and 2c). Moreover, the levels of the pro-inflammatory cytokines IL-6, IL-12/ IL-23p40 and TNF- $a$ were significantly elevated in BALF of the $\mathrm{Mfge8}^{-/}$mice, whereas the levels of the antiinflammatory cytokine TGF- $\beta 1$ were also markedly increased (Figure 1f).

MFG-E8 modulates early peritoneal inflammation after pristane treatment. The numbers of PMNs and macrophages in the peritoneum of the $\mathrm{Mfge}^{-/-}$and WT mice were quantified on day 0 , at $16 \mathrm{~h}$, and on days 5,10 and 14 (Figure 2a). The Mfge $^{-/-}$mice showed an increase in total leukocytes in the peritoneum at $16 \mathrm{~h}$ and day 5; the leukocytes were mainly composed of $\mathrm{CD}_{11} \mathrm{~b}^{+}$cells (Figure 2b). As early as $16 \mathrm{~h}$, the number of PMNs in the peritoneal cavity was significantly elevated, and continued to accumulate until day 14, accompanied by leukocyte infiltration into the lung (Figure 1d). Moreover, macrophage recruitment was markedly reduced at $16 \mathrm{~h}$ and 5 and 10 days in the $\mathrm{Mfge}^{-1-}$ mice (Figure $2 \mathrm{~b}$ ). In line with peritoneal PMN accumulation and decrease in the number of macrophages, the levels of pro-inflammatory cytokines, such as IL-1 $\beta$, IL-6 and IL-12/IL-23p40, were significantly increased after pristane treatment for 5 days, whereas the TNF-a levels were obviously elevated on day 10 . However, the levels of these cytokines were decreased on day 14 in the $\mathrm{Mfge}^{-1-}$ mice. In parallel, a decrease in the anti-inflammatory TGF- $\beta 1$ and IL-10 levels on day 5 and day 10 was observed in the $\mathrm{Mfge}^{-1}$ mice compared with the WT mice (Figure 2c). Autoantibody production in the pristane-injected mice was dependent on IFN-I, which was primarily secreted by $\mathrm{Ly}^{+} \mathrm{C}^{+}$ immature monocytes in the pristane-induced lupus. ${ }^{3,7,25}$ The results revealed that monocytes were significantly increased on day 14 after pristane treatment, and the IFN- $a$ levels were markedly increased in the $\mathrm{Mfge}^{-/}$mice compared with the WT mice (Figure 2d).

MFG-E8 deficiency aggravates neutrophil recruitment in pristane-induced lupus. Differentially labeled $\mathrm{Mfge}^{-/}$and WT bone marrow-derived neutrophils (BMDNs) were equally i.v. injected into one mouse that was pre-treated with pristane to further validate the neutrophil migration observed in lung and peritoneum. The result showed that a markedly larger number of $\mathrm{Mfge8}^{-/}$BMDNs migrated into the peritoneum and lung in vivo compared with the WT BMDNs (Figure 3a). Moreover, in vitro, the number of migrated $\mathrm{Mfge}^{-/-}$BMDNs was increased compared with the WT BMDNs with or without MIP-2 stimulation (Figure 3b). After pretreatment with 

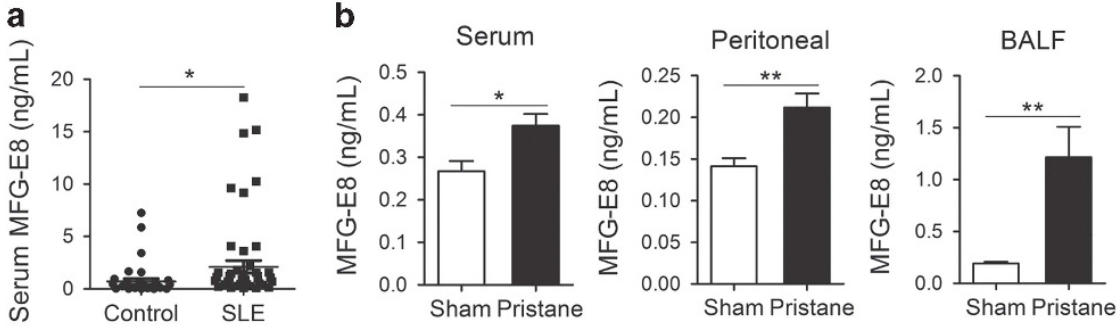

C
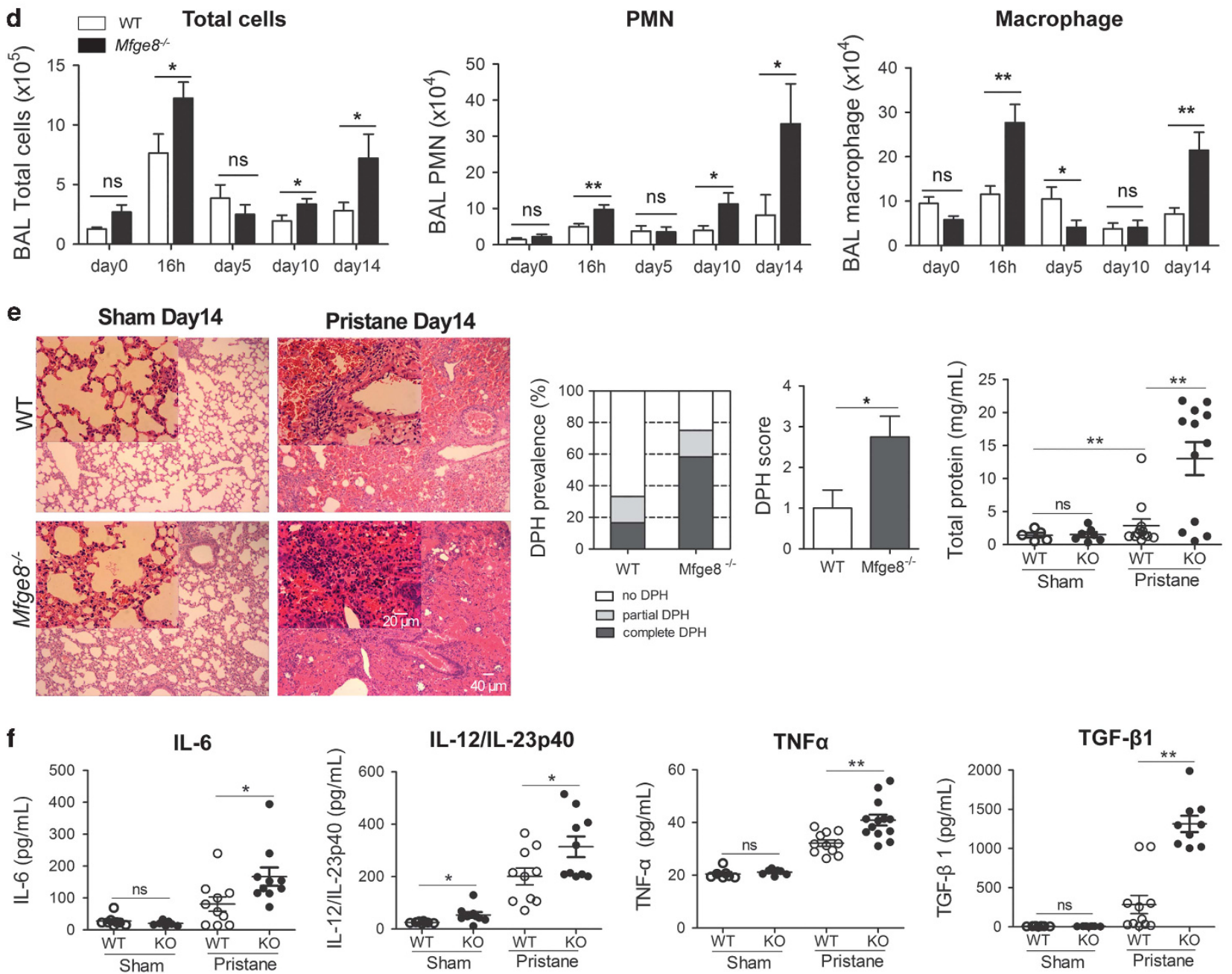

Figure 1 MFG-E8 is essential for human SLE and mice with pristane-induced lupus. (a) The serum MFG-E8 concentration from 35 healthy controls and 51 active SLE patients were measured by ELISA. (b) After a 24-week pristane exposure, the serum levels of MFG-E8 in WT mice were detected by ELISA; MFG-E8 concentrations in BALF and peritoneal cavity of WT mice exposed to pristane for 2 weeks were determined by ELISA ( $n=10-12$ mice per group). (c) Western blotting of MFG-E8 expression in the lung tissues of WT mice injected PBS or pristane for 2 weeks. Mouse MFG-E8 was detected as $~ 68$ and $~ 58$ kD protein in SDS-PAGE. (d) Quantified BAL total cells, BAL PMNs and macrophages at $0 \mathrm{~h}, 16 \mathrm{~h}$, and on days 5,10 and 14 by flow cytometer analysis ( $n=8-12$ mice per group). (e) Pathology of the lung tissues from Mfge $8^{-/-}$mice and WT mice with H\&E staining, each group of which was injected PBS or pristane for 14 days, were shown $(n=10-12$ mice per group). Representative images were displayed, original magnification $\times 100$ and $\times 400$. Prevalence of DPH and DPH score were estimated based on H\&E staining; total proteins in BALF were detected with ELISA. (f) The cytokines IL-6, IL-12/IL-23p40, TNF- $\alpha$ and TGF- $\beta 1$ concentrations in BAL were determined with ELISA on day 14 after pristane injection ( $n=10-12$ mice per group). For all experiments, data are presented as means \pm S.E.M., ${ }^{*} P<0.05,{ }^{\star \star} P<0.01$; ns, not significant

rmMFG-E8, the numbers of neutrophils were significantly reduced in the peritoneal cavities and lungs from WT or $\mathrm{Mfge}^{-/-}$mice compared with the mice that were not treated with rmMFG-E8 after being exposed to pristane for $16 \mathrm{~h}$ (Figure 3c). In addition, the numbers of migrated $\mathrm{Mfge}^{-/-}$ and WT BMDNs in vitro were markedly decreased after the
rmMFG-E8 treatment compared with the BMDNs without rmMFG-E8 treatment when exogenous MIP-2 was added as a chemotactic stimulus (Figure 3d).

The secretion of MIP-2, a critical chemokine responsible for neutrophil chemotaxis, was markedly increased after pristane exposure; however, there was no significant difference in the 
MIP-2 levels in the BALF and peritoneum of the $\mathrm{Mfges}^{-/-}$and WT mice (Supplementary Figure 3a). Intriguingly, CXCR2 is the receptor expressed on neutrophil for MIP-2-dependent chemotaxis, $^{26}$ and its expression on BMDNs from the $\mathrm{Mfge}^{-/}$mice was $\sim 40 \%$ higher than WT BMDNs (Figure $3 e$ ). An increased in the neutrophil ratio was apparent

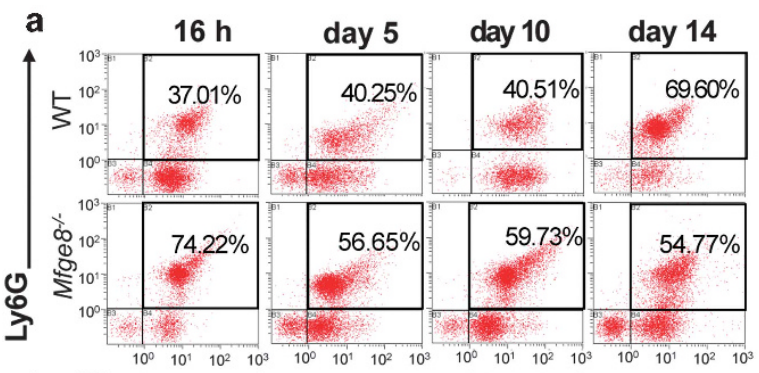

b

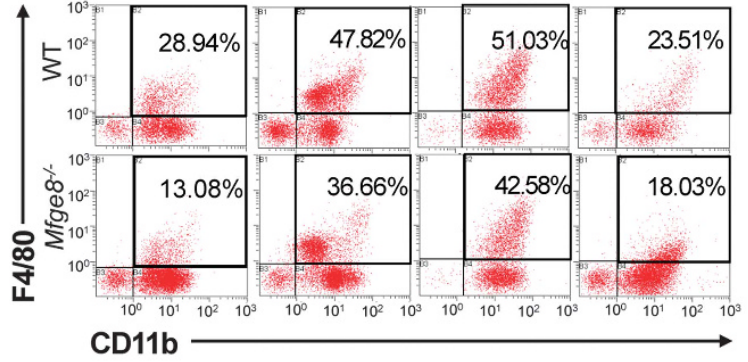

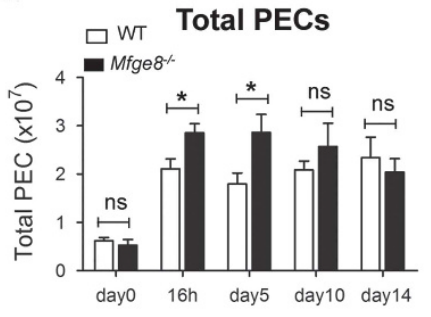

PMN
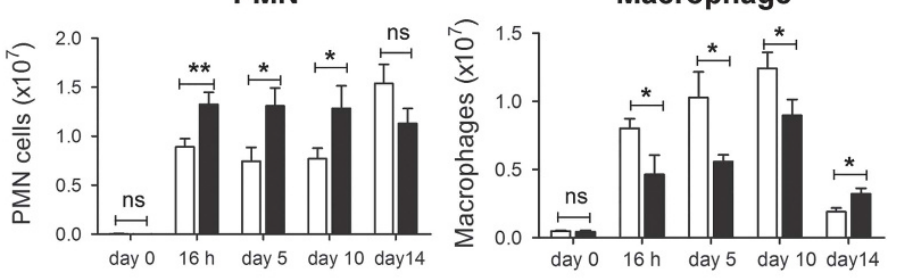
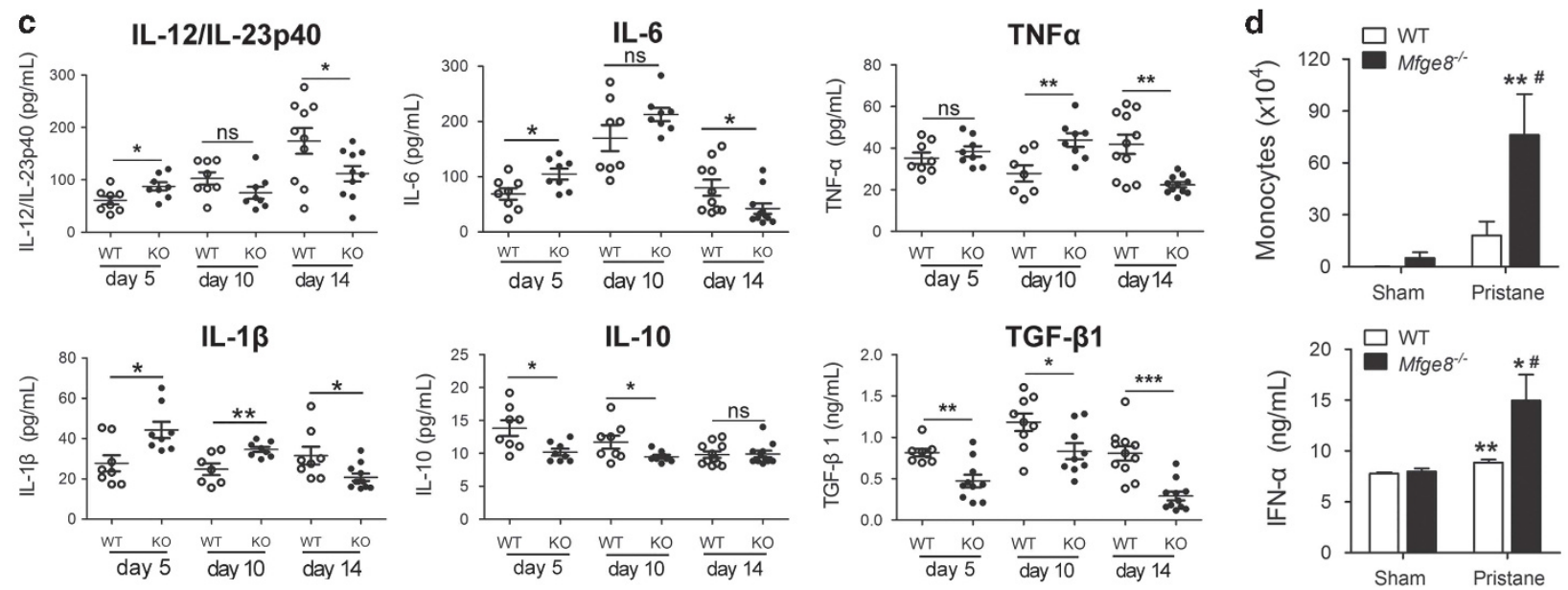

Figure 2 MFG-E8 deficiency enhances the early peritoneal inflammatory response to pristane. (a) Definition of peritoneal PMNs and macrophages subsets by flow cytometry, gating for $\mathrm{CD} 11 \mathrm{~b}^{+} \mathrm{F} 4 / 80^{+}$macrophages, $\mathrm{CD} 11 \mathrm{~b}^{+} \mathrm{Ly} 6 \mathrm{G}^{+} \mathrm{PMNs}$ in the peritoneum and representative images at $16 \mathrm{~h}$, and on days 5,10 and 14 after pristane treatment were shown. (b) Statistical analysis of total cells, CD11 $\mathrm{b}^{+}$cells, PMNs and macrophages at $0 \mathrm{~h}, 16 \mathrm{~h}$, and days 5,10 and 14 ( $n=8-12$ mice per group). (c) Peritoneal cytokine profiles response to pristane. IL-12/LL-23p40, IL-6, TNF- $\alpha$, IL-1 $\beta$, TGF- $\beta 1$ and IL-10 concentrations were determined by ELISA on days 5,10 and 14 ( $n=8-12$ mice per group). For all experiments, data are presented as means \pm S.E.M., ${ }^{*} P<0.05$, ${ }^{\star *} P<0.01$; ns, not significant. (d) Quantified peritoneal Ly6C ${ }^{+}$monocytes from $M f g e 8^{-/-}$mice and WT mice on day 14 with or without pristane injection by flow cytometry, and detected IFN- $\alpha$ levels on day 14 with ELISA. Data are presented as means \pm S.E.M., ${ }^{*} P<0.05$, ${ }^{* \star} P<0.01$ versus PBS treatment, ${ }^{\#} P<0.05$ versus WT with pristane treatment

Figure 3 Effects of MFG-E8 on neutrophil recruitment in mice with pristane-induced lupus and human SLE. (a) BMDNs tracking assay. Recruited PKH67 ${ }^{+}$Mfge $8^{-1}$ BMDNs and PKH26 ${ }^{+}$WT BMDNs into lung and peritoneum were detected by flow cytometry ( $n=6$ mice per group). (b) Mfge $8^{-1-}$ and WT BMDNs for migration assay in vitro with or without rmMIP-2 $(1 \mathrm{ng} / \mathrm{ml})$ stimulation. Representative images of migrated BMDNs were shown, original magnification $\times 100$. More than five random microscopic fields per well were counted. (c) In pristane-induced early inflammation (16 h), after pretreatment with rmMFG-E8 (20 $\mu \mathrm{g} / \mathrm{kg})$, infiltrated PMN in the lung and peritoneum from Mfge $8^{-/-}$and WT mice were determined by flow cytometry ( $n=6-8$ mice per group). (d) Mfge $8^{-/}$and WT BMDNs for migration assay in vitro with or without rmMFG-E8 (500 ng/ml) treatment, when rmMIP-2 $(1 \mathrm{ng} / \mathrm{ml})$ was added as chemotactic stimulus, were determined. (e) The surface expression of CXCR2 on Mfge ${ }^{-1-}$ and WT BMDNs were analyzed by flow cytometry, and the average mean fluorescence intensities (MFI) for CXCR2 were shown. (f) PMN ratio in PBCs and their surface CXCR2 expression from 36 healthy controls and 52 SLE patients were measured by flow cytometry and the average MFI for CXCR2 were calculated. (g) After treatment with $500 \mathrm{ng} / \mathrm{ml}$ rhMFG-E8, surface expression of CXCR2 on isolated neutrophils from SLE patients were determined ( $n=12$ individuals per group). (h) After pretreatment with rmMFG-E8 (20 $\mu \mathrm{g} / \mathrm{kg})$, surface expression of CXCR2 on BMDNs from WT mice, which were exposed to pristane for $16 \mathrm{~h}$ were detected by flow cytometry ( $n=6$ mice per group). For all experiments, data are presented as means $\pm S$.E. M., ${ }^{*} P<0.05,{ }^{* \star} P<0.01,{ }^{* \star} P<0.001$; ns, not significant 
in the peripheral blood cells (PBCs) of SLE patients compared with the healthy controls, but the plasma chemokine IL-8 levels were decreased in the SLE patients (Supplementary Figure $3 b)$. Therefore, we examined the expression of surface CXCR2 and found that an $\sim 18 \%$ increase in its expression on neutrophils from SLE patients compared with the healthy controls (Figure 3f). Furthermore, the recombinant human MFG-E8 (rhMFG-E8) treatment induced a significant downregulation of CXCR2 expression on the neutrophils from SLE patients in vitro (Figure $3 \mathrm{~g}$ ). In pristane-treated WT mice, the surface expression of CXCR2 on neutrophils in bone marrow was greatly enhanced compared with untreated mice, and the

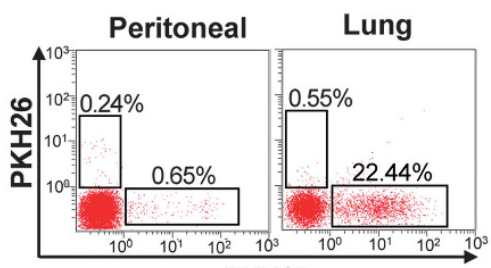

PKH67
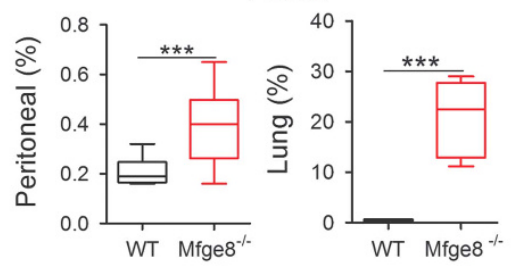

C

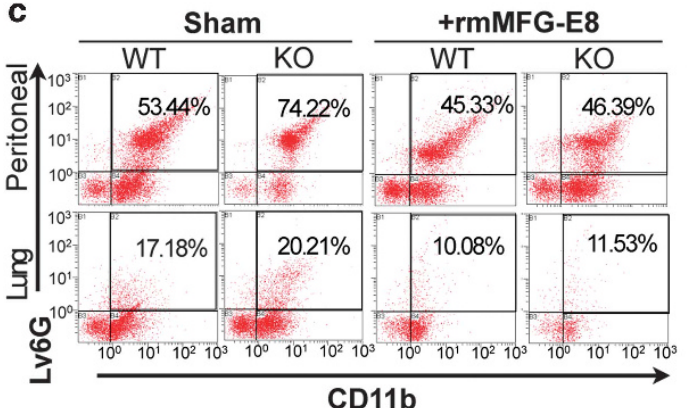

b
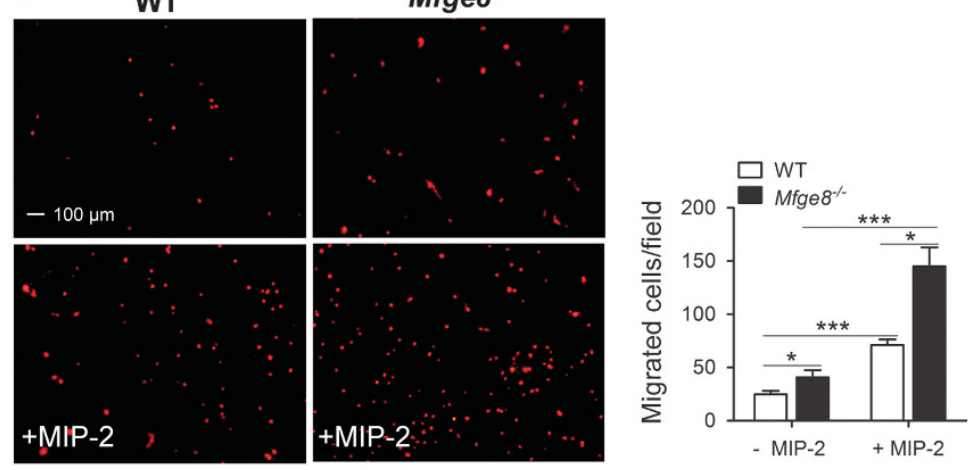

d
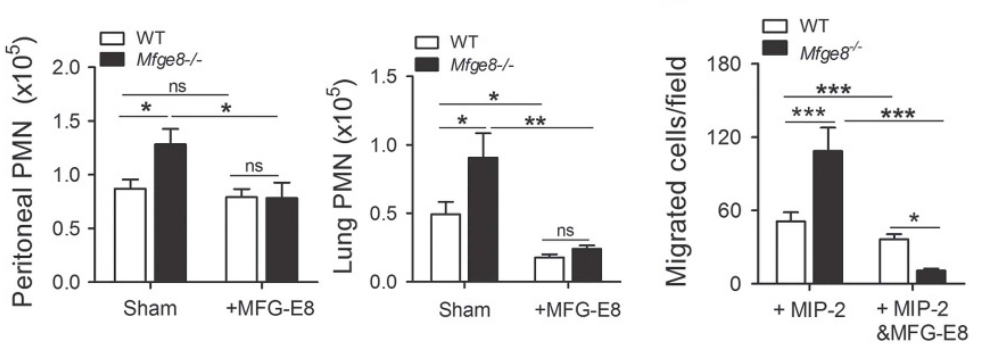

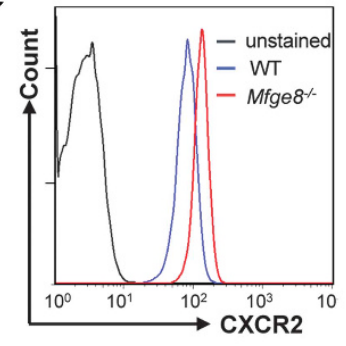

$f$

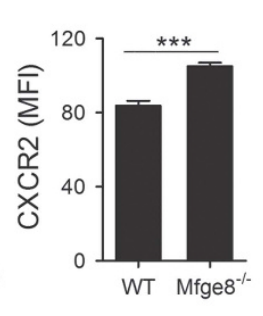

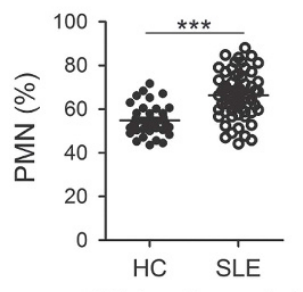
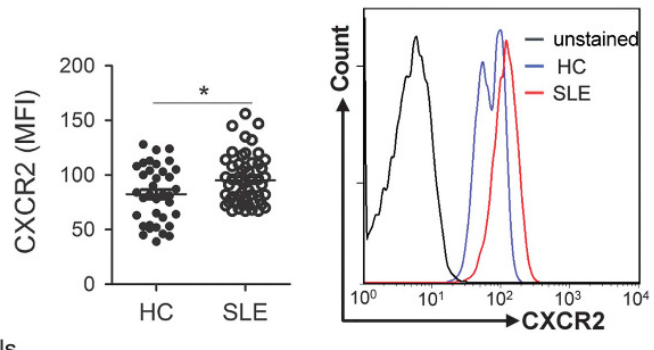

g

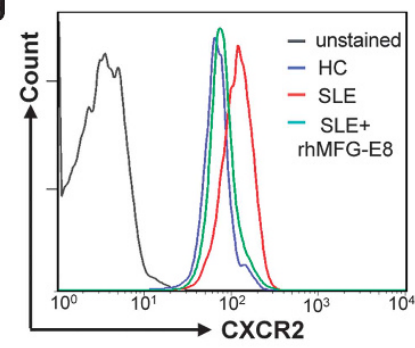

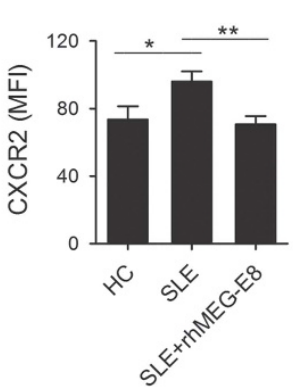

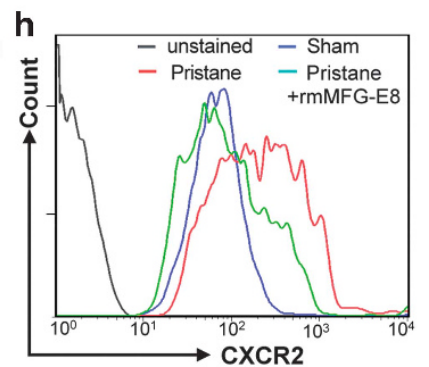

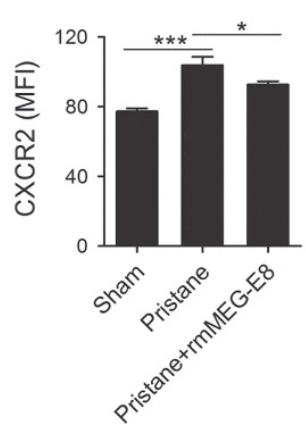


a

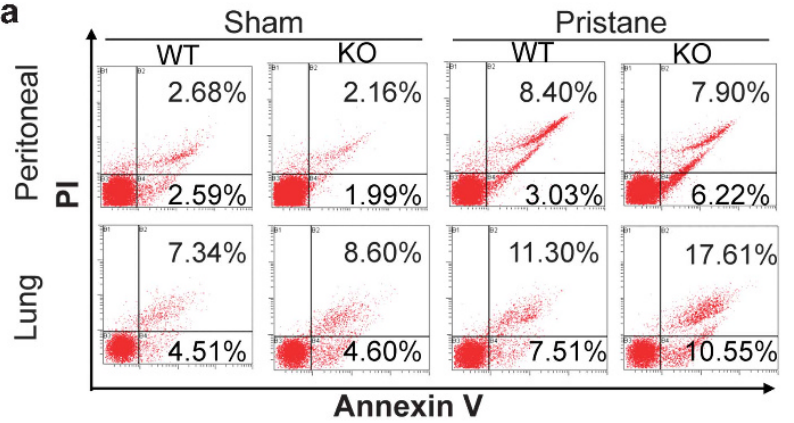

b
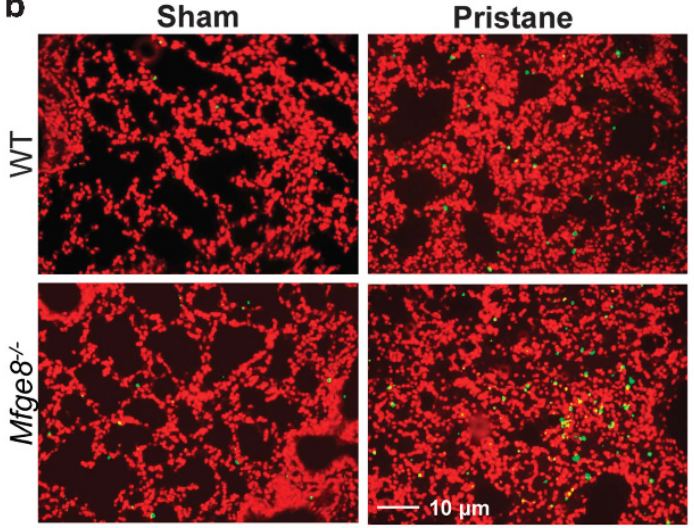
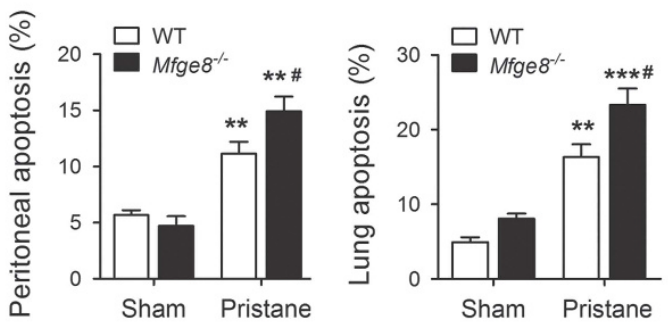

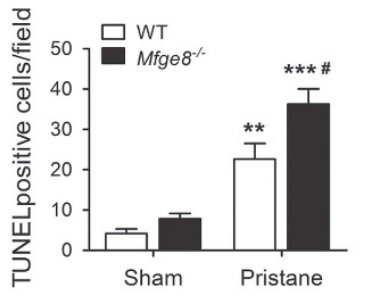

C $\quad$ WT $\frac{\text { Mfge8 } \%}{--+++}$

$\mathrm{Bcl}-2-\cdots \cdots-\cdots+27 \mathrm{KD}$

$\mathrm{Bcl}-\mathrm{xL}-\infty \ldots \ldots-\infty 29 \mathrm{KD}$

$\mathrm{Bax}=--\ldots 20 \mathrm{KD}$

$\beta$-actin $-43 \mathrm{KD}$

MFG-E8 $\quad \mathbb{2} \mathbb{E E} \ldots \ldots 8 \mathrm{KD}$

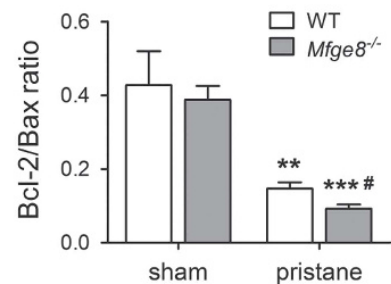

d

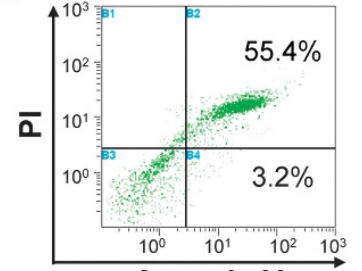

Annexin V e

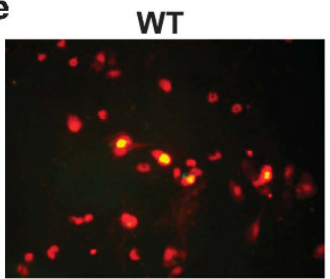

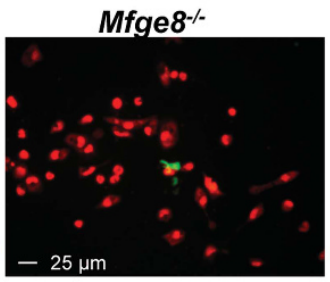

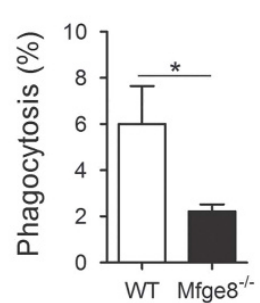

Figure 4 MFG-E8 deficiency leads to accumulation of death cells. (a) Apoptotic leukocytes in BALF and peritoneum of Mfge $8^{-1-}$ and WT mice on 5 days after pristane exposure were stained with Annexin V/PI and determined by flow cytometry ( $n=6-8$ mice per group). (b) Infiltration of death cells into the Mfge8 ${ }^{-/-}$and WT lungs after a 2-week pristane exposure was identified. Representative images were shown, original magnification $\times 200$. The average TUNEL-positive cells were counted at more than five random microscopic fields. (c) For further estimate apoptosis, the lung tissues were subjected to western blotting to detect MFG-E8, Bcl-2, Bcl-xL and Bax protein levels. Mouse Bcl-2, $\mathrm{Bcl}-\mathrm{xL}$ and Bax were detected as $\sim 27, \sim 29$ and $\sim 20 \mathrm{kD}$ protein, respectively, in SDS-PAGE. Results were normalized with $\beta$-actin, and Bcl-2/Bax ratios were evaluated and displayed. Data are presented as means \pm S.E.M., ${ }^{\star *} P<0.01,{ }^{* \star} P<0.001$ versus PBS treatment; ${ }^{\sharp} P<0.05$ versus WT with pristane treatment. (d) Apoptotic BMDNs were stained with Annexin V/PI and detected by flow cytometry. (e) Peritoneal macrophages from the Mfge $8^{-/}$mice showed impairment of phagocytosis. Isolated peritoneal macrophages from Mfge8 ${ }^{-/-}$and WT mice were stained with PI and apoptotic BMDNs were stained with green fluorescence PKH67. Representative images were shown, original magnification $\times 400$. The phagocytic index was evaluated by phagocytotic macrophages/total macrophages per field. The average phagocytosis was counted at more than five random microscopic fields. Data are means \pm S.E.M. from more than two independent experiments, ${ }^{*} P<0.05$

upregulation of CXCR2 was abolished by pretreatment with rmMFG-E8 (Figure 3h). Although serum MFG-E8 levels were significantly elevated in active SLE patients and mice with pristane-induced lupus (Figures $1 \mathrm{a}$ and $\mathrm{b}$ ), these increased MFG-E8 were not enough to downregulate the surface expression of CXCR2 on neutrophil, according to its dosedependent downregulation. ${ }^{21,22}$

Accumulation of apoptotic cells in pristane-induced inflammation. Pristane-induced apoptotic cells were the critical source of autoantigens and the initial event in the pathogenesis of lupus. ${ }^{27}$ The proportions of early apoptotic and late apoptotic/necrotic cells were significantly increased in the BALF and peritoneum of $\mathrm{Mfge}^{-1-}$ mice compared with WT mice after pristane injection (Figure 4a). After pristane treatment for 2 weeks, a marked increase in the number of dead cells trapped in lung tissue of $\mathrm{Mfges}^{-/}$mice was observed compared with WT mice (Figure 4b). Moreover, the $\mathrm{Bcl}-2 / \mathrm{Bax}$ ratio was significantly decreased in the $\mathrm{Mfge8}^{-/}$ lung tissues compared with the WT tissues (Figure 4c). The reason for the aggravated accumulation of dead cells in lung and peritoneum was partially caused by the increased neutrophil infiltration and abnormal apoptosis in the $\mathrm{Mfges}^{-/}$mice and partially was caused by the inefficient 
a

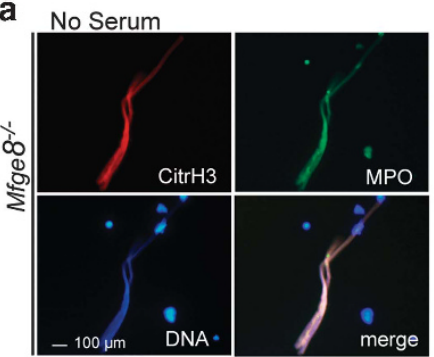

b

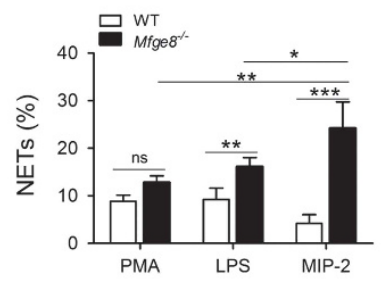

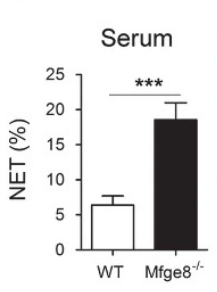

d
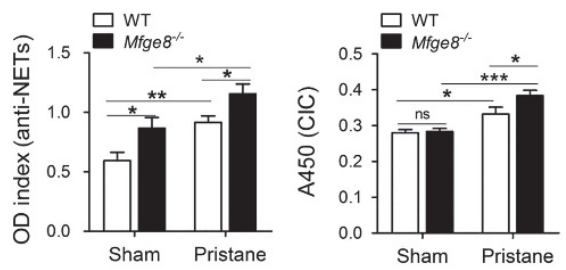

e
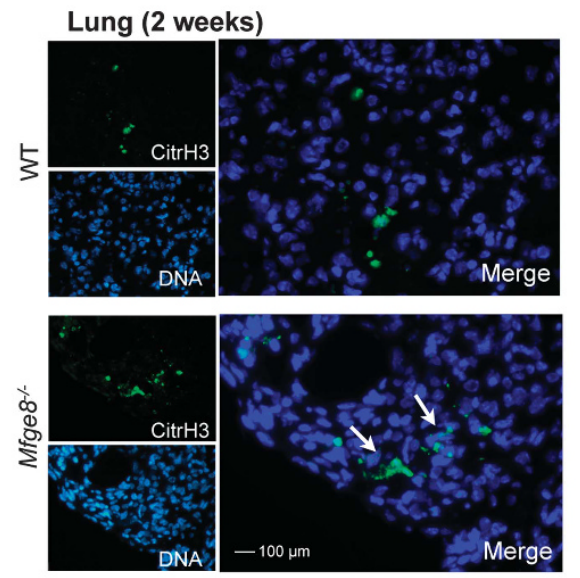
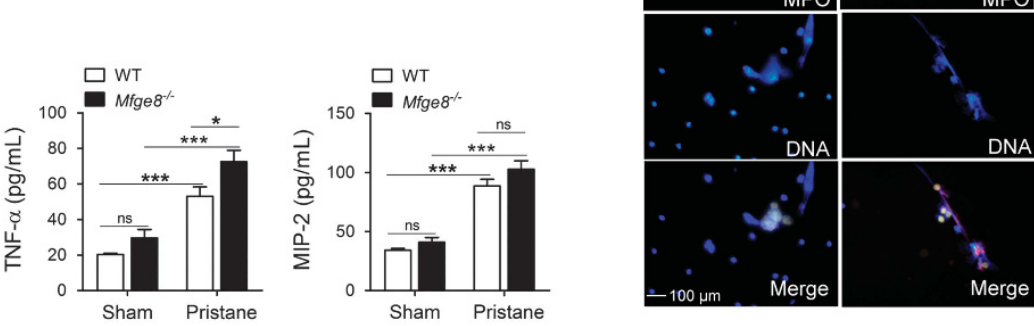

f

Lung (24 weeks)
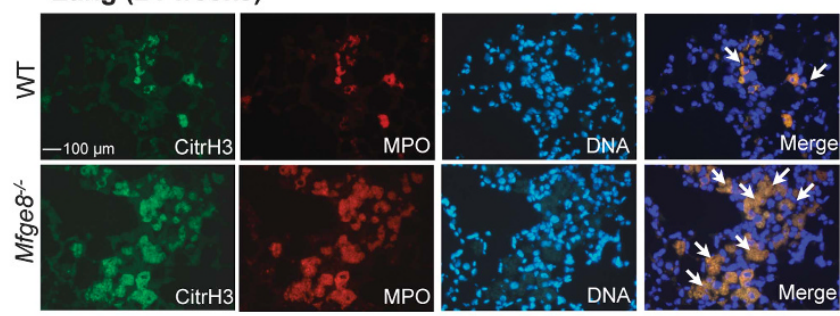

g

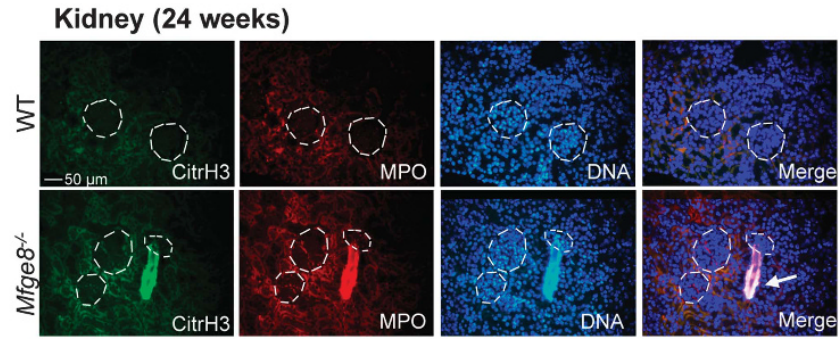

Figure 5 MFG-E8-deficient neutrophils were primed to NETs release. (a) BMDNs from the Mfge8 ${ }^{-1-}$ or WT mice spontaneously release NETs without serum cultivation. Representative fluorescence microscopic images showing NETs. DNA was stained blue, citrullinated histone H3 was stained green and MPO was stained red. Three colors were merged by software Image $\mathrm{J}$ (National Institutes of Health, Bethesda, MD, USA), original magnification $\times 400$. NET\% = positive enlarged nuclei/total neutrophils per field. The average of NET release ratio was evaluated $>500$ cells in more than five fields per sample were counted. (b) PMA, LPS and MIP-2 treatment induced NETs formation from the Mfge $^{-/-}$and WT BMDNs. (c) The serum from pristane-treated mice stimulated NETs release. WT BMDNs were incubated with $2 \%$ serum isolated from the Mfge ${ }^{-/}$or WT, which were exposed to pristane for 24 weeks mice. Representative fluorescence microscopic images showing NETs release. (d) The serum levels of anti-NET antibodies, CICs, TNF- $\alpha$ and MIP-2 in Mfge8 ${ }^{-/-}$and WT mice after pristane exposure for 24 weeks were measured by ELISA. (e) In vivo evidence for NETs formation in the lung tissues of Mfge8 ${ }^{-1}$ - mice after pristane exposure for 2 weeks. NETs were identified by close localization of DNA (blue) with citrullinated histone H3 (green), white arrows indicated NETs. (f) NETs were visualized in the lung tissues of $\mathrm{Mfge}^{-/-}$or WT mice after pristane exposure for 24 weeks. (g) NETs were located around glomeruli in kidney tissues of Mfge8 $^{-/-}$mice after treatment with pristane for 24 weeks. Enlarged nuclei were founded to be citrullinated histone H3 and MPO positive, white arrows indicated NETs. For all experiments, data are shown as the means \pm S.E.M., ${ }^{*} P<0.05,{ }^{* \star} P<0.01,{ }^{* \star} P<0.001$; ns, not significant

phagocytosis of apoptotic cells by the $\mathrm{Mfge8}^{-/-}$macrophages. Indeed, the peritoneal $\mathrm{Mfge}^{-1}$ macrophages showed impairments in phagocytosing the apoptotic neutrophils, which were induced with serum starvation (Figures $4 d$ and e) and phosphatidylserine (PS)-coated carboxylated beads (Supplementary Figure 4).

MFG-E8-deficient neutrophils are primed to release NETs. NETosis is the main source of SLE-derived antiribonucleoprotein antibodies and IFN-I production. ${ }^{7,28}$
Neutrophils isolated from bone marrow of $\mathrm{Mfge8}^{-/}$mice showed significantly enhanced spontaneous neutrophil extracellular trap (NET) formation without serum cultivation compared with the WT mice (Figure 5a). When phorbol 12-myristate 13-acetate (PMA), LPS or the chemokine MIP-2 were added as stimuli, there was no significant difference in NET release between the $M f g e 8^{-1-}$ and WT BMDNs after stimulation with PMA; however, the $\mathrm{Mfge}^{-/}$BMDNs revealed higher NET formation following stimulation with LPS or MIP-2 (Figure 5b; Supplementary Figure 5). Among 
a

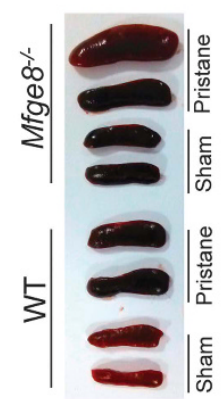

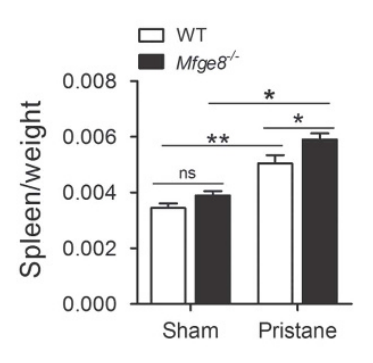

c

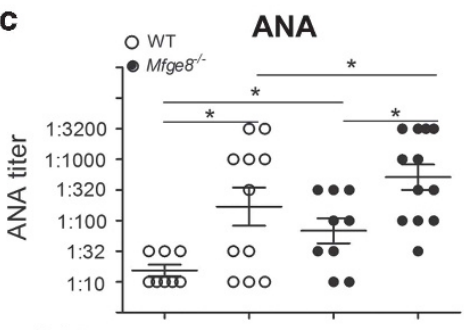

Pristane

e

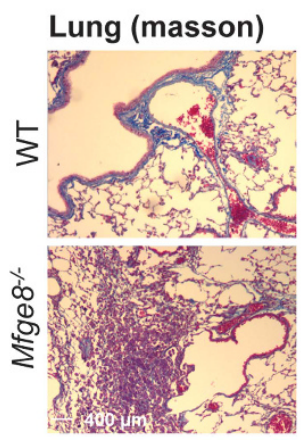

f
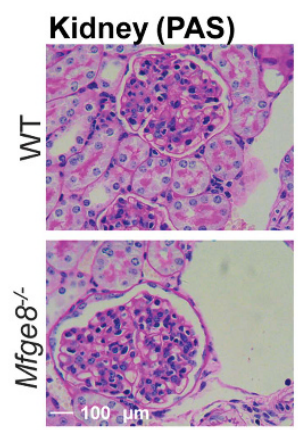

g

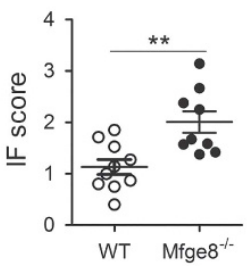

\section{b Spleen (H\&E)}

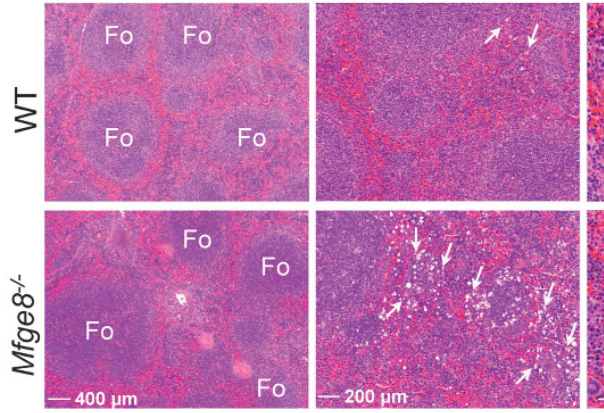

Oil droplets

d

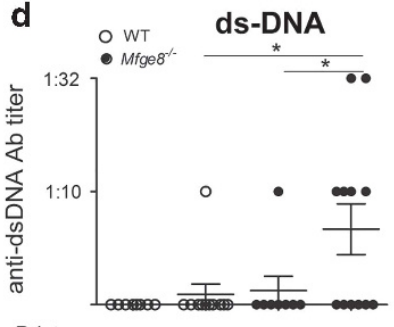

Pristane

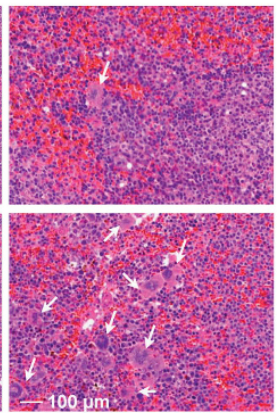

megakaryocytes

ANCA

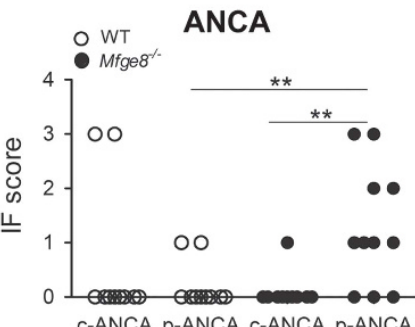

c-ANCA p-ANCA c-ANCA p-ANCA
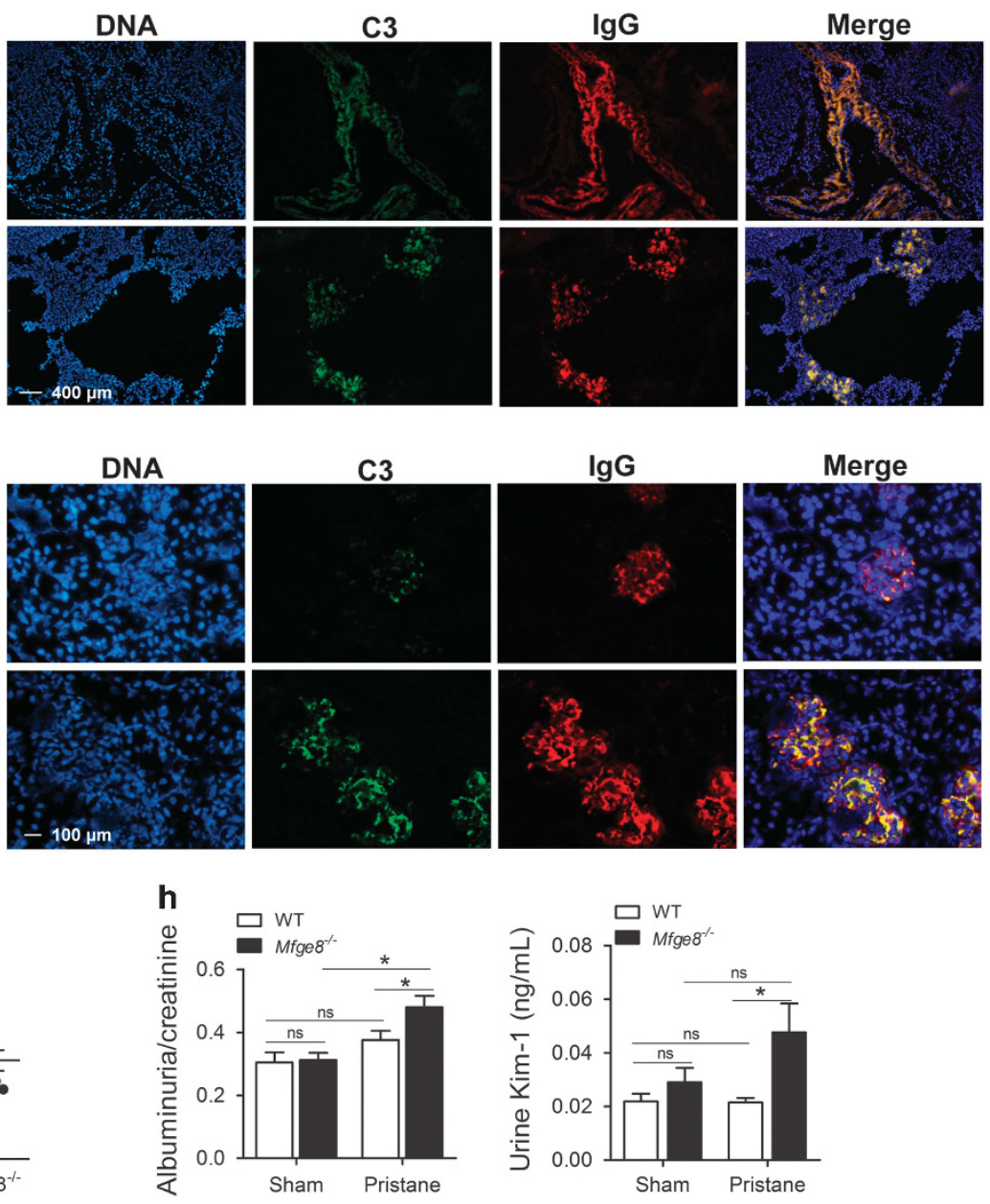
these three stimuli, the $M f g e 8^{-/-}$BMDNs exhibited markedly increased NET release after stimulation with MIP-2, which may be dependent on the enhanced expression of CXCR2 on the surface of $\mathrm{Mfge}^{-/-}$neutrophils (Figure $5 \mathrm{~b}$ ). NET-derived materials ultimately induced the production of autoantibodies, such as anti-dsDNA and anti-NET antibodies. In contrast, these antibodies prevented DNase I from accessing and dismantling the NETs. ${ }^{9,29,30}$ A variety of stimuli could trigger NETosis, including pathogens, pro-inflammatory cytokines (IL-8 and TNF-a), PMA, nitric oxide (NO), anti-neutrophil cytoplasmic antibodies (ANCAs), immune complexes, etc. ${ }^{4}$ In this study, compared with the serum from WT mice, the serum from pristane-treated $\mathrm{Mfges}^{-/}$mice induced increased NET formation (Figure 5c), owing to the increase in the CICs, TNF- $a$ levels, a trend toward increased MIP-2 levels, and anti-NET antibodies, which combined with NETs to avoid being degraded in the serum of the $\mathrm{Mfge}^{-/-}$mice (Figure $5 d$ ). Furthermore, the NETs were lodged in areas of lung tissue with hemorrhagic lesions following early exposure to pristane (Figure 5e), and NETs were predominantly located within the alveoli of the $\mathrm{Mfge}^{-/-}$mice with pulmonary complications of extensive interstitial consolidation after pristane exposure for 24 weeks (Figure 5f). Similarly, NETs were formed in the kidney tissues around the glomeruli in the $\mathrm{Mfge}^{-/-}$mice that were treated with pristane for 24 weeks (Figure 5g).

The lack of MFG-E8 exacerbates the induction of autoantibody production and immune complex deposition following pristane exposure. After 24 weeks, splenomegaly was observed in the $M f g e 8^{-/}$mice treated with or without pristane (Figure 6a); in parallel, obvious pathological features were visualized, including enlarged B-cell follicles (white pulps), the infiltration of oil droplets and increased numbers of megakaryocytes (Figure 6b). These abnormalities were also aggravated in the lung and liver of the pristane-treated $\mathrm{Mfge}^{-/-}$mice. H\&E staining revealed that the bronchial wall was markedly thickened, ectopic lymphoid cells were abnormally accumulated around the bronchus and hemosiderin-laden macrophages were deposited within the alveoli (Supplementary Figure 6a); similarly, there was an increase in the number of neutrophils, oil droplets, ectopic lymphoid cells or lipogranulomas in the liver (Supplementary Figure $6 \mathrm{~b}$ ). Pristane exposure induced the production of antinuclear antibody (ANA), and $\mathrm{Mfge8}^{-/}$mice showed higher serum ANA levels than the WT mice at 24 weeks (Figure 6c). Weak anti-dsDNA antibodies were produced in the serum of WT mice (1/11 mice, 9.1\%); however, serum anti-dsDNA antibody production was markedly elevated in the $\mathrm{Mfge}^{-/-}$ mice (6/12 mice, 50\%) after the 24-week pristane treatment (Figure 6d). Furthermore, the serum of $\mathrm{Mfges}^{-/}$mice revealed higher ANCA levels than WT mice. There was also an effect on the autoantibody specificity: pristane-injected WT mice frequently generated an antibody that commonly presented as a c-ANCA antibody (proteinase 3-ANCA, PR3-ANCA); in contrast, most of the pristane-injected Mfge $^{-/}$mice predominantly exhibited p-ANCA antibodies (myeloperoxidase-ANCA (MPO-ANCA)) (Figure 6d, Supplementary Figure 7).

After pristane exposure for 24 weeks, increased infiltration with collagen fibers and interstitial hemosiderin-laden macrophages were in the interstitial alveoli of the $\mathrm{Mfge}^{-/-}$mice, whereas collagen fibers were infused around the blood vessels in the WT mice (Figure 6e). In addition, the lack of MFG-E8 resulted in the deposition immune complexes within the interstitial alveoli, but the immune complexes deposited within blood vessels of WT lung tissue (Figure 6e). In the kidney, the extent of glomerular cellularity and glomerular immune complexes were significantly increased in the Mfge $^{-1}$ - mice compared with the WT mice (Figures $6 f$ and g). Moreover, the pristane-treated $\mathrm{Mfges}^{-/}$mice showed a marked enhancement in the albuminuria/creatinine ratio and a trend toward increased Kim-1 levels (Figure 6h).

\section{Discussion}

It has been reported that the number of tingible body macrophages, which usually contain engulfed apoptotic nuclei, was significantly reduced in SLE patients, leading to apoptotic cells not being properly cleared in germinal centers and activating complement and functioning as a survival signal for B cells that autoimmunity could arise..$^{31,32}$ The adhesion and phagocytosis of apoptotic cell material by monocytederived macrophages were also impaired in patients with SLE. ${ }^{33,34}$ Furthermore, differentiated macrophages showed decreased phagocytosis of apoptotic neutrophils because of the lower levels of genes involved apopto-phagocytosis, such as C1QA, C2, GAS6 and MFG-E8 etc. ${ }^{35}$ Pristane induced apoptosis of lymphoid cells both in vivo and in vitro, which was a critical initiating event in the pathogenesis of pristaneinduced lupus and was of potential relevance for human SLE. ${ }^{27}$ Although the murine lupus model did not completely simulate human SLE, there were several similarities between SLE and murine lupus, particularly in the pristane-induced lupus model (Supplementary Table 1). MFG-E8-deficient mice were utilized as a murine lupus model and developed

Figure 6 MFG-E8 effects autoantibody production and immune complex deposition. (a) Splenomegaly was visualized in the spleens from pristane-treated ${\text { Mfge } 8^{-/}}^{/ 2}$ mice, and spleen/weight ratios in mice with pristane-induced lupus were calculated $\left(n=8-12\right.$ mice per group). (b) Histopathology of the spleens from pristane-treated Mfge $^{-/-}$and WT mice; Fo = B-cell follicle. White arrows indicated infiltrated oil droplets or megakaryocytes. (c) The serum ANA titers from Mfge $8^{-/}$and WT mice with pristane exposure for 24 weeks were detected by immunofluorescence ( $n=8-12$ mice per group). (d) The serum anti-dsDNA antibody titers and ANCA in the Mfge8 ${ }^{-/-}$and WT mice after a 24-week pristane exposure were determined by immunofluorescence ( $n=8-12$ mice per group); the proportion of c-ANCA and p-ANCA was calculated. (e) Left, with Masson-trichrome staining, histopathology of the lungs from Mfge $8^{-/-}$and WT mice after exposure to pristane for 24 weeks were shown; right, deposition of C3 (green) and lgG (red) in the lungs from $\mathrm{Mfge}^{-1-}$ and WT mice were displayed. (f) Left, the histopathology of renal tissues from the Mfge $8^{-1-}$ and WT mice exposed to pristane for 24 weeks were shown with PAS staining; right, deposition of C3 (green) and lgG (red) in glomeuli of kidney tissues from the Mfge $8^{-/}$and WT mice were shown. (g) Composite scores of C3 and lgG were averaged and shown. (h) Left, albuminuria and creatinine in urine were detected by ELISA ( $n=8-12$ mice per group), and albuminuria-to-creatinine ratios were calculated; right, the Kim-1 levels in urine were examined with ELISA ( $n=8-12$ mice per group). For all experiments, data are shown as means $\pm S . E . M .,{ }^{*} P<0.05,{ }^{* *} P<0.01 ; n s$, not significant 
splenomegaly as a result of the impaired removal of the apoptotic B cells by tingible body macrophages and follicular dendritic cells in the germinal centers, leading to autoimmune disease. ${ }^{14,36}$

In recent years, the initial innate immunity has inspired new value in studying development of organ damage in the kidney, the vasculature, the skin and other tissues in SLE. ${ }^{25,37}$ One proposed mechanism that contributes to autoantibody production in pristane-induced lupus is an increased burden of apoptotic neutrophils, which may either overexpress autoantigens on their cell surface by aggregation, or release them because of inefficient phagocytosis, leading to necrosis. In this study, MFG-E8-deficient mice revealed increased neutrophil infiltration and apoptosis, or even necrosis because of impaired phagocytosis of Mfge $^{-/-}$macrophages. The other proposed mechanism to enhance NETosis, resulting in increased autoantigens exposure and autoantibody production. The pristane-treated MFG-E8-deficient mice enhanced NETs formation caused by increased stimuli including CICs, cytokines and neutrophil susceptibility with overexpressed surface CXCR2. Taken together, MFG-E8 affects the phenotype and function of neutrophils to ameliorate their abnormal features and decreases neutrophil death through apoptosis and NETosis.

Although MFG-E8 expression was upregulated in the lung and peritoneal cavity of mice developing pristane-induced lupus, it marked the disease severity because MFG-E8 was highly expressed in inflammatory macrophages (M1 macrophages) but not anti-inflammatory macrophages (M2 macrophages) at the inflamed sites. ${ }^{38}$ In WT mice, a comparable number of inflammatory macrophages were recruited to the peritoneum, which expressed MFG-E8 and promoted the phagocytosis of dead cells, such as apoptotic neutrophils. In contrast, the phagocytosis of apoptotic neutrophils promoted the polarization of M2 macrophages, anti-inflammatory subset, to inhibit the development and progression of SLE. ${ }^{23} \mathrm{We}$ speculated that the impaired phagocytosis of the apoptotic neutrophils by recruited macrophages in peritoneal cavity revealed the decreased polarization of M2 macrophages in Mfge $^{-/}$mice, in line with increased pro-inflammatory cytokine secretion compared with the WT mice.

With the end result of capillaritis exclusively in the lung, the histopathological features of DPH include capillary wall necrosis with infiltration by leukocytes, interstitial erythrocytes or hemosiderosis. ${ }^{39}$ In the early phase of DPH without pulmonary immune complex deposition, other inflammatory factors, such as infection or drugs trigger the pulmonary injury, which cause oxidative stress, and may be a trigger for the development of fibrosing interstitial pneumonias. ${ }^{24,39}$ Collagen fiber accumulation was also visualized in the interstitial alveoli of the MFG-E8-deficient mice with autoantibody production in pristane-induced lupus, indicating that MFG-E8 may indirectly attenuate pulmonary fibrosis by ameliorating the early DPH in the experimental lupus model.

Implicit in the current model of SLE pathogenesis was that extracellular DNA was derived from the apoptotic debris by an aberrant apoptotic process. However, recent studies indicated that extracellular DNA may frequently be released by NETosis, which stimulated with pathogens, pro-inflammatory cytokines (IL-8 and TNF-a), PMA, NO, ANCAs, immune complex and circulating apoptotic microparticles etc. ${ }^{4,40}$ In pristane-induced murine model, the serum MIP-2 and TNF- $a$ levels were elevated, particularly in $M f g e 8^{-1-}$ mice, which may stimulate neutrophils to release NETs. Although the detailed signaling pathways for NET formation remain elusive, it was believed that the generation of reactive oxygen species (ROS) by nicotinamide adenine dinucleotide phosphate (NADPH) oxidase was indispensable, ${ }^{41}$ and peptidylarginine deiminase 4 , citrullinated histones and nucleosomes, had crucial roles in chromatin decondensation to form the NETs. ${ }^{9,42}$ Immobilized immune complexes induced NET formation from primary human neutrophils, and its intracellular signaling pathways were involved in the tyrosine kinase Src/Syk pathway. ${ }^{43}$ In pristanetreated $\mathrm{Mfge}^{-/-}$mice, the amount of CICs was significantly increased, and more immune complexes were lodged in the lung and kidney, which contributed to trigger the release of NETs. Further, Lood and colleague's ${ }^{44}$ study highlighted an important role of mitochondrial ROS in immune complexmediated NETosis, which oxidized the mtDNA and promoted NET release in SLE patients. In addition, ANCAs activated neutrophils to release ROS and pro-inflammatory cytokines, which could stimulated neutrophil to release NETs and expose autoantigens MPO and PR3. ${ }^{45-47}$ In our findings, serum from the MFG-E8-deficient mice that were exposed to pristane showed increased levels of the MPO-ANCA compared with WT mice. Furthermore, the higher titer of MPO-ANCA production in the $\mathrm{Mfge}^{-/}$mice may also contribute to higher prevalence of DPH, because several reports demonstrated that MPO-ANCAassociated vasculitides were related with $\mathrm{DPH}$ and renal injury. ${ }^{48,49}$ The dysregulation of NETosis in the $\mathrm{Mfges}^{-1}$ mice not only involved in the overproduction of NETs but also a decrease in NET degradation. The endonuclease DNase I was responsible for NET degradation, and the impaired NET degradation was related to the increased anti-NET and antidsDNA antibody titers. ${ }^{4,30}$ The perpetuation of non-degraded NETs may lead to the production of anti-NET antibodies and complement system activation, which in turn block NET degradation, forming a vicious circle. In our studies, the increased NET formation in the pristane-treated $\mathrm{Mfge}^{-/}$mice was also explained by the result that $\mathrm{Mfge}^{-/}$mice possessed higher anti-NET antibodies and anti-dsDNA antibodies titer in serum.

In SLE or pristane-induced murine lupus, neutrophils infiltrated into the inflamed sites, dependent on the upregulation of surface receptor CXCR2, which was attenuated by exogenous MFG-E8. However, CXCR2 not only modulated neutrophil recruitment but also mediated NET formation independent of NADPH oxidase and involved Src family kinases. $^{29}$ Rac2, a small Rho GTPase, is essential for CXCR2-mediated NET release induced by IL-8, through the $\mathrm{PI} 3 \mathrm{~K}$ and Src-ELMO-Dock2 pathways involving ROS and NO. ${ }^{50,51}$ In our findings, the Mfge $^{-/-}$neutrophils, expressed high levels of CXCR2, increased NET release upon MIP-2 stimulation. Therefore, Rac2 may have a central role in NET formation induced by the chemokine MIP-2 and CXCR2mediated signaling pathway in the pristane-induced murine model, and future studies are required further explore and systematically determine these possibilities. Taken together, MFG-E8 had an important role in mediating surface CXCR2 expression to suppress neutrophil migration and NET release. 
In summary, we highlighted the role of neutrophils in the pathogenesis of SLE and found that MFG-E8 had a crucial role in attenuating the aberrant responses of neutrophils, as its absence resulted in a significant upregulation of dead cells, NET formation, the type-I IFN response, autoantibody synthesis and glomerulonephritis. Through its regulatory function in neutrophil, MFG-E8 may represent a therapeutic target to control early inflammation to efficiently treat environmental exposure lupus.

\section{Materials and Methods}

Patients. Sera from 51 active treated or non-treated young female (20-45 years old) SLE patients and 35 healthy individuals who were admitted to Wuhan No.1 Hospital and diagnosed with SLE according to the American College of Rheumatology revised criteria ${ }^{52}$ were used to detect the MFG-E8 levels. Meanwhile, EDTA-anticoagulated PBCs from 52 active, treated or non-treated female SLE patients and 36 healthy individuals were applied to determine the CXCR2 receptor expression levels on neutrophils. Clinical and serological information was obtained from the patients' medical records. This study was approved by the ethics committee of Wuhan No. 1 Hospital (Wuhan, China).

Experimental model. Mfge $8^{-/}$mice were constructed in a C57BL/6j background by deleting an exon III to exon IIV genomic fragment generated by Nanjing Biomedical Research Institute of Nanjing University (NBRI, Nanjing, China) and were maintained under specific pathogen-free conditions at the animal housing facility at Wuhan No.1 Hospital. For lupus induction, female $\mathrm{Mfge}^{-/-}$mice and agematched wild-type (WT) littermates received a single $0.5 \mathrm{ml}$ i.p. injection of pristane (Sigma-Aldrich, St. Louis, MO, USA) or phosphate buffered saline (PBS) as a control at 8-12 weeks of age. The recombinant MFG-E8 (R\&D Systems, Minneapolis, MN, USA) was i.p. administered at a dose of $20 \mu \mathrm{g} / \mathrm{kg}$ body weight $2 \mathrm{~h}$ before the i.p. pristane injection. All protocols were approved by the Institutional Animal Care and Use Committee of the Wuhan No.1 Hospital.

\begin{abstract}
Histopathology, TUNEL assay and immune complex deposition. Lung, spleen and liver tissues were fixed with formalin, embedded in paraffin and sectioned. Then, the tissue sections were stained with H\&E or Masson's-trichrome stain. The prevalence of lungs with hemorrhage was estimated and the score of the left lobe was calculated using H\&E staining: 4, 75-100\%; 3, 50-75\%; 2, 25-50\%; 1 , $0-25 \%$; and 0 , no hemorrhage. ${ }^{23}$ For the in situ TUNEL assay, cell death was determined using a TUNEL staining kit (Roche Diagnostics, Indianapolis, IN, USA). ${ }^{21}$ Briefly, sections of paraffin-embedded lung tissues were deparaffinized and subjected to antigen retrieval. Then, the sections were incubated with a mixture of $\mathrm{TdT}$ and fluorescence-labeled nucleotides. The prepared sections were examined with Olympus BX51 microscope (Olympus, Tokyo, Japan) and Qimaging camera (RoHs, Surrey, British Columbia, Canada). For pulmonary immune complex deposition, the frozen sections were stained with FITC-conjugated anti-C3 and Alexa Fluor 647-conjugated anti-mouse IgG (Abcam, Cambridge, UK). DNA was visualized using DAPI (Sigma-Aldrich), and the sections were examined with Olympus BX51 microscope and Qimaging camera.
\end{abstract}

Western blot analysis. Proteins from the lung tissues were separated by SDS-PAGE, and the MFG-E8 protein was detected with a goat anti-mouse MFG-E8 antibody (R\&D Systems). The lung proteins were incubated with rabbit anti-Bcl-2 $m A b$, anti-Bcl-XL $m A b$, anti-Bax $m A b$ and anti- $\beta$-actin $m A b$ (Cell Signaling Technology, Danvers, MA, USA) and then visualized with an HRP-conjugated secondary antibody (Southern Biotech, UAB, Birmingham, AL, USA) and chemilumiescence (Thermo Pierce, Rockford, IL, USA) to further detect apoptosis.

Flow cytometry analysis. Leukocyte subsets in the BALF and peritoneal cavity were stained with following mAbs: FITC-labeled anti-CD11b (BD Biosciences, Franklin Lakes, NJ, USA), PE-labeled Ly6G (BD Biosciences), PE-cyanine 5-labeled F4/80 (eBiosciences, San Diego, CA, USA) and PE-cy 7-labeled Ly6C (BD Biosciences). Neutrophils in the PBCs from SLE patients and healthy controls were stained with PE-CD16b (BD Biosciences). Dead cells in the BALF and peritoneal cavity were stained with PI and FITC-labeled Annexin V according to Apoptosis Detection Kit I (BD Pharmingen, San Diego, CA, USA). BMDNs from WT, $\mathrm{Mfge}^{-1-}$ mice, WT mice that were exposed to pristane for $16 \mathrm{~h}$, or WT mice that were exposure with pristane before the rmMFG-E8 injection were stained with the FITC-CD11b, PE-Ly6G and PerCP/Cy5.5-CD182 (CXCR2) mAbs (Biolegend, San Diego, CA, USA), respectively, and subjected to flow cytometry analysis (Beckman Coulter, Fullerton, CA, USA) to examine the surface expression of CXCR2 on murine neutrophils. The CXCR2 level was identified in the $\mathrm{CD}_{11 b^{+}} \mathrm{Ly}_{6 \mathrm{G}}{ }^{+}$cells. PBCs from SLE patients or healthy controls were stained with mAbs PE-CD16b and FITC-CD182 (CXCR2) (BD Biosciences), and subjected to flow cytometry analysis to determine the CXCR2 expression levels on human neutrophils, and the CXCR2

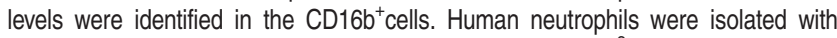
Percoll (Sigma-Aldrich) gradients (65 and $75 \%$ ), and $1.5 \times 10^{6}$ cells were incubated with $500 \mathrm{ng} / \mathrm{ml} \mathrm{rhMFG}-\mathrm{E} 8$ (R\&D Systems) in RPMI- 1640 medium for $2 \mathrm{~h}$ to test the effect of MFG-E8 on CXCR2 on human neutrophils. The data were analyzed by FlowJo software (Tree Star, Ashland, OR, USA), with 10000 events per sample.

In vivo and in vitro neutrophil migration assay. WT and Mfge $8^{-/}$neutrophils were isolated from bone marrow cells using Percoll (SigmaAldrich) gradients $(55,62$ and $81 \%$ Percoll in PBS). Cells at the $62 / 81 \%$ interface were extracted and identified by flow cytometry. BMDNs isolated from the WT and Mfge $8^{-1}$ mice were labeled with fluorescent PKH26 and PKH67 (Sigma-Aldrich), respectively, according to the manufacturer's instructions. The labeled WT and Mfge $8^{-1-}$ BMDNs were i.v. injected together in equal numbers into WT mice $1 \mathrm{~h}$ after pristane treatment, and $16 \mathrm{~h}$ later, the migration of the transfused BMDNs was observed in the peritoneal cavity and lung by flow cytometry. The migration was conducted in Transwell polycarbonate inserts $(6.5 \mathrm{~mm}$ diameter) with 3- $\mu \mathrm{m}$-poresize membranes (Costa, Cambridge, MA, USA) in vitro with or without treatment with $1 \mathrm{ng} / \mathrm{ml}$ recombinant murine (rm) MIP-2 (R\&D Systems). After a 2-h incubation, the migrated cells were fixed with formalin and stained with PI (Sigma-Aldrich). The rmMFG-E8 protein $(500 \mathrm{ng} / \mathrm{ml})$ (R\&D Systems) was added along with rmMIP-2 $(1 \mathrm{ng} / \mathrm{ml})$ as the stimulus to explore the role MFG-E8 in neutrophil migration.

Phagocytosis. Isolated WT BMDNs were cultured in RPMI-1640 medium without serum in $5 \% \mathrm{CO}_{2}$ at $37^{\circ} \mathrm{C}$ for $20 \mathrm{~h}$. Apoptotic neutrophils were detected by flow cytometry with Apoptosis Detection Kit I (BD Pharmingen) and labeled with PKH67 (Sigma-Aldrich). Macrophages were harvested from the peritoneum of WT or $\mathrm{Mfge}^{-1-}$ mice $72 \mathrm{~h}$ after the injection of $0.5 \mathrm{ml}$ pristane (Sigma-Aldrich). After an 18-h incubation, the non-adherent WT or Mfge $8^{-1-}$ macrophages were removed and the apoptotic neutrophils were added in the culture at ratio of $1: 3$ (macrophages:neutrophils). After a 4-h cultivation, the non-phagocytotic neutrophils were washed out, and the macrophages were fixed and stained with PI.

Neutrophil extracellular traps. Identification of NETs in this study was similar to a previously published protocol. ${ }^{10}$ Briefly, $2 \times 10^{5}$ BMDNs were seeded onto poly-L-lysine-coated coverslips (Sigma-Aldrich). In experiments without stimulation or with stimulation with $2 \%$ serum (extracted from pristane-treated WT or Mfge $8^{-/-}$mice for 6 months), the cells were incubated for $2 \mathrm{~h}$. In experiments with $1 \mu \mathrm{g} / \mathrm{ml}$ LPS, $100 \mathrm{nM}$ PMA or $100 \mathrm{ng} / \mathrm{ml}$ MIP-2 stimulation, the incubation was for $4 \mathrm{~h}$. NETs were stained with rabbit anti-histone H3 (citrulline R2+R8+R17) antibody and mouse anti-MPO antibody, followed by incubation with Alexa Fluor 647-conjugated mouse IgG and Alexa Fluor 488-conjugated rabbit IgG (Abcam). DNA was stained with DAPI. Images were collected with Olympus BX51 microscope and Qimaging camera, typically at original $\times 400$ magnification.

ELISA. The concentrations of mouse MFG-E8 (R\&D Systems), IL-1 $\beta$ (eBiosciences), TNF- $\alpha$ (eBiosciences), IL-6 (eBiosciences), IL-12/IL-23p40 (R\&D Systems), TGF- $\beta 1$ (R\&D Systems), IL-10 (R\&D Systems) and MIP-2 (R\&D Systems) in the BALF, peritoneal lavage fluid and serum were detected using ELISA kits. The levels of mouse CICs were determined with ELISA kits (Alpha Diagnostic, San Antonio, TX, USA). The Kim-1 (R\&D Systems), albumin level in urine collected during the last $24 \mathrm{~h}$ of the experiments was determined by ELISA. The serum levels of anti-NET antibodies were detected with an in-house ELISA. ${ }^{9}$ Briefly, NET protein was prepared by stimulating $\mathrm{Mfge}^{-/}$bone marrow neutrophils with $100 \mathrm{nM}$ PMA for $12 \mathrm{~h}$, collecting the supernatants and precipitating the protein with $80 \%$ acetone. In all, $100 \mathrm{ng} / \mathrm{ml}$ NET proteins were coated on 96-well high-binding EIA/RIA plates overnight. After blocking, mouse serum that had been diluted $1: 100$ was added to the plates and incubated for $2 \mathrm{~h}$; then, HRP-conjugated anti-mouse IgG (Southern Biotech) was added at a 1:8000 dilution. The serum MFG-E8 levels (CUSABIO, Wuhan, China) and plasma IL-8 concentrations (R\&D Systems) in SLE patients and healthy controls were determined using human ELISA kits. 
Sarcosine oxidase enzymatic (SOE) assay. Creatinine $(\mathrm{Cr})$ in urine collected during the last $24 \mathrm{~h}$ of the experiments was determined by SOE assay. Urine $\mathrm{Cr}$ was quantified by Beckman enzymatic $\mathrm{Cr}$ reagent/Beckman AU5800 biochemical analyzer (Beckman Coulter, Fullerton, CA, USA). The Beckman alkaline picrate $\mathrm{Cr}$ reagent was also evaluated on the Beckman AU5800 analyzer for comparison. Thus, the renal injury was expressed using the Kim-1 level and the albumin: $\mathrm{Cr}$ ratio.

Glomerulonephritis. Formalin-fixed, paraffin-embedded and Periodic acidSchiff (PAS)-stained kidney sections were assessed for glomerular pathology. ${ }^{53}$ For glomerular immune complex deposition, IgG and C3 were quantified in frozen sections, as previously described. ${ }^{54}$ At least 20 glomeruli per animal were assessed by two observers in blinded manner to determine the average scores of the immune complexes. Scores on a scale of 0-3 were estimated for both $\mathrm{C} 3$ and $\mathrm{IgG}$ deposition, based on the fluorescence intensity, and each component (IgG and C3) was added to create composite average score.

Determination of autoantibodies. Serum ANA, anti-dsDNA antibody and ANCA levels were determined by immunofluorescence (Euroimmun, Lübeck, Germany). The serum was diluted $1: 10,1: 100$ and $1: 1000$, to determine the ANA titer. Crithidia luciliae was utilized as the antigen substrate and the serum was diluted 1:10 to detect anti-dsDNA antibody. The ANCA titer was estimated in serum diluted $1: 10$ using primate liver, Hep2 cells, formalin-fixed neutrophils and ethanol-fixed neutrophils as substrates. Then, FITC-conjugated mouse IgG secondary antibody (Southern Biotech) was added, and the images were captured on EUROStar III plus (Euroimmun) at $\times 200$ or $\times 400$ magnification.

Statistical analysis. Results are reported as the means \pm S.E.M. A two-tailed Student's $t$-test was used to determined the significance of the differences using SPSS 16.0 (Chicago, IL, USA) or GraphPad Prism software 5.0 (GraphPad, La Jolla, CA, USA). A $P$-value of $<0.05$ was considered statistically significant.

\section{Conflict of Interest}

The authors declare no conflict of interest.

Acknowledgements. We are grateful to Nanjing Biomedical Research Institute of Nanjing University (NBRI, China) for constructing Mfge $8^{-/}$mice. This work was supported by grants of National Natural Science Foundation of China (no. 81170035 and no. 81502222), and Wuhan Huanghe Talent program (2012).

\section{Author contributions}

WH, TPC and DYY conceived and designed experiments. WH, YX and RJ performed experiments. WH, JYW and HQYanalyzed the data. WH wrote the manuscript, and all authors discussed the results and commented on the manuscript.

1. Azevedo PC, Murphy G, Isenberg DA. Pathology of systemic lupus erythematosus: the challenges ahead. Methods Mol Biol 2014; 1134: 1-16.

2. Dorner T, Jacobi AM, Lee J, Lipsky PE. Abnormalities of B cell subsets in patients with systemic lupus erythematosus. J Immunol Methods 2011; 363: 187-197.

3. Denny MF, Yalavarthi S, Zhao W, Thacker SG, Anderson M, Sandy AR et al. A distinct subset of proinflammatory neutrophils isolated from patients with systemic lupus erythematosus induces vascular damage and synthesizes type I IFNs. J Immunol 2010; 184: 3284-3297.

4. Yu Y, Su K. Neutrophil extracellular traps and systemic lupus erythematosus. J Clin Cell Immunol 2013; 4: 139.

5. Wu SA, Yeh KW, Lee WI, Yao TC, Kuo ML, Huang B et al. Impaired phagocytosis and susceptibility to infection in pediatric-onset systemic lupus erythematosus. Lupus 2013; 22: 279-288.

6. Midgley A, McLaren Z, Moots RJ, Edwards SW, Beresford MW. The role of neutrophil apoptosis in juvenile-onset systemic lupus erythematosus. Arthritis Rheum 2009; 60: 2390-2401.

7. Garcia-Romo GS, Caielli S, Vega B, Connolly J, Allantaz F, Xu Z et al. Netting neutrophils are major inducers of type I IFN production in pediatric systemic lupus erythematosus. SCi Transl Med 2011; 3: 73ra20.

8. Mahajan A, Herrmann M, Munoz LE. Clearance deficiency and cell death pathways: a model for the pathogenesis of SLE. Front Immunol 2016; 7: 35.

9. Knight JS, Zhao W, Luo W, Subramanian V, O'Dell AA, Yalavarthi S et al. Peptidylarginine deiminase inhibition is immunomodulatory and vasculoprotective in murine lupus. J Clin Invest 2013; 123: 2981-2993.
10. Villanueva E, Yalavarthi S, Berthier CC, Hodgin JB, Khandpur R, Lin AM et al. Netting neutrophils induce endothelial damage, infiltrate tissues, and expose immunostimulatory molecules in systemic lupus erythematosus. J Immunol 2011; 187: $538-552$.

11. Hanayama R, Tanaka M, Miwa K, Shinohara A, Iwamatsu A, Nagata S. Identification of a factor that links apoptotic cells to phagocytes. Nature 2002; 417: 182-187.

12. Aziz M, Jacob A, Matsuda A, Wang P. Review: milk fat globule-EGF factor 8 expression, function and plausible signal transduction in resolving inflammation. Apoptosis 2011; 16 : 1077-1086.

13. Miyasaka K, Hanayama R, Tanaka M, Nagata S. Expression of milk fat globule epidermal growth factor 8 in immature dendritic cells for engulfment of apoptotic cells. Eur J Immunol 2004; 34: 1414-1422.

14. Hanayama R, Tanaka M, Miyasaka K, Aozasa K, Koike M, Uchiyama Y et al. Autoimmune disease and impaired uptake of apoptotic cells in MFG-E8-deficient mice. Science 2004; 304: 1147-1150.

15. Scaffidi P, Misteli T, Bianchi ME. Release of chromatin protein HMGB1 by necrotic cells triggers inflammation. Nature 2002; 418: 191-195.

16. Yamaguchi H, Takagi J, Miyamae T, Yokota S, Fujimoto T, Nakamura S et al. Milk fat globule EGF factor 8 in the serum of human patients of systemic lupus erythematosus. J Leukoc Biol 2008; 83: 1300-1307.

17. Yamamoto $\mathrm{N}$, Yamaguchi $\mathrm{H}$, Ohmura K, Yokoyama T, Yoshifuji $\mathrm{H}$, Yukawa $\mathrm{N}$ et al. Serum milk fat globule epidermal growth factor 8 elevation may subdivide systemic lupus erythematosus into two pathophysiologically distinct subsets. Lupus 2014; 23: 386-394.

18. Peng Y, Elkon KB. Autoimmunity in MFG-E8-deficient mice is associated with altered trafficking and enhanced cross-presentation of apoptotic cell antigens. J Clin Invest 2011; 121: 2221-2241.

19. Hu CY, Wu CS, Tsai HF, Chang SK, Tsai WI, Hsu PN. Genetic polymorphism in milk fat globule-EGF factor 8 (MFG-E8) is associated with systemic lupus erythematosus in human. Lupus 2009; 18: 676-681.

20. Cui T, Miksa M, Wu R, Komura H, Zhou M, Dong W et al. Milk fat globule epidermal growth factor 8 attenuates acute lung injury in mice after intestinal ischemia and reperfusion. Am J Respir Crit Care Med 2010; 181: 238-246.

21. Aziz M, Matsuda A, Yang WL, Jacob A, Wang P. Milk fat globule-epidermal growth factor-factor 8 attenuates neutrophil infiltration in acute lung injury via modulation of CXCR2. J Immunol 2012; 189: 393-402.

22. Aziz M, Yang WL, Corbo LM, Chaung WW, Matsuo S, Wang P. MFG-E8 inhibits neutrophil migration through alphavbeta(3)-integrin-dependent MAP kinase activation. Int $J$ Mol Med 2015; 36: 18-28.

23. Shi $\mathrm{Y}$, Tsuboi N, Furuhashi K, Du Q, Horinouchi A, Maeda $\mathrm{K}$ et al. Pristane-induced granulocyte recruitment promotes phenotypic conversion of macrophages and protects against diffuse pulmonary hemorrhage in Mac-1 deficiency. J Immunol 2014; 193: 5129-5139.

24. Chowdhary VR, Grande JP, Luthra HS, David CS. Characterization of haemorrhagic pulmonary capillaritis: another manifestation of Pristane-induced lupus. Rheumatology (Oxford) 2007; 46: 1405-1410.

25. Denny MF, Thacker S, Mehta H, Somers EC, Dodick T, Barrat FJ et al. Interferon-alpha promotes abnormal vasculogenesis in lupus: a potential pathway for premature atherosclerosis. Blood 2007; 110: 2907-2915.

26. Olson TS, Ley K. Chemokines and chemokine receptors in leukocyte trafficking. Am J Physiol Regul Integr Comp Physiol 2002; 283: R7-28.

27. Calvani N, Caricchio R, Tucci M, Sobel ES, Silvestris F, Tartaglia P et al. Induction of apoptosis by the hydrocarbon oil pristane: implications for pristane-induced lupus. J Immunol 2005: 175: 4777-4782.

28. Herman S, Kny A, Schorn C, Pfatschbacher J, Niederreiter B, Herrmann M et al. Cell death and cytokine production induced by autoimmunogenic hydrocarbon oils. Autoimmunity 2012; 45: 602-611.

29. Marcos V, Zhou Z, Yildirim AO, Bohla A, Hector A, Vitkov L et al. CXCR2 mediates NADPH oxidase-independent neutrophil extracellular trap formation in cystic fibrosis airway inflammation. Nat Med 2010; 16: 1018-1023.

30. Hakkim A, Furnrohr BG, Amann K, Laube B, Abed UA, Brinkmann V et al. Impairment of neutrophil extracellular trap degradation is associated with lupus nephritis. Proc Natl Acad Sci USA 2010; 107: 9813-9818.

31. Munoz LE, Lauber K, Schiller M, Manfredi AA, Herrmann M. The role of defective clearance of apoptotic cells in systemic autoimmunity. Nat Rev Rheumatol 2010; 6: 280-289.

32. Baumann I, Kolowos W, Voll RE, Manger B, Gaipl U, Neuhuber WL et al. Impaired uptake of apoptotic cells into tingible body macrophages in germinal centers of patients with systemic lupus erythematosus. Arthritis Rheum 2002; 46: 191-201.

33. Herrmann M, Voll RE, Zoller OM, Hagenhofer M, Ponner BB, Kalden JR. Impaired phagocytosis of apoptotic cell material by monocyte-derived macrophages from patients with systemic lupus erythematosus. Arthritis Rheum 1998; 41: 1241-1250.

34. Tas SW, Quartier P, Botto M, Fossati-Jimack L. Macrophages from patients with SLE and rheumatoid arthritis have defective adhesion in vitro, while only SLE macrophages have impaired uptake of apoptotic cells. Ann Rheum Dis 2006; 65: 216-221.

35. Majai G, Kiss E, Tarr T, Zahuczky G, Hartman Z, Szegedi G et al. Decreased apopto-phagocytic gene expression in the macrophages of systemic lupus erythematosus patients. Lupus 2014; 23: 133-145. 
36. Kranich J, Krautler NJ, Heinen E, Polymenidou M, Bridel C, Schildknecht A et al. Follicular dendritic cells control engulfment of apoptotic bodies by secreting Mfge8. J Exp Med 2008; 205: 1293-1302.

37. Denny MF, Chandaroy P, Killen PD, Caricchio R, Lewis EE, Richardson BC et al. Accelerated macrophage apoptosis induces autoantibody formation and organ damage in systemic lupus erythematosus. J Immunol 2006; 176: 2095-2104.

38. Hanayama R, Tanaka M, Miwa K, Nagata S. Expression of developmental endothelial locus-1 in a subset of macrophages for engulfment of apoptotic cells. J Immunol 2004; 172 : 3876-3882.

39. Cohen S. Diffuse pulmonary hemorrhage: evolutionary 'flaw' or consequence of evolutionary progress? Am J Med Sci 2002; 323: 130-139.

40. Dieker J, Tel J, Pieterse E, Thielen A, Rother N, Bakker $M$ et al. Circulating apoptotic microparticles in systemic lupus erythematosus patients drive the activation of dendritic cell subsets and prime neutrophils for NETosis. Arthritis Rheumatol 2016; 68: 462-472.

41. Fuchs TA, Abed U, Goosmann C, Hurwitz R, Schulze I, Wahn V et al. Novel cell death program leads to neutrophil extracellular traps. J Cell Biol 2007; 176: 231-241.

42. Li P, Li M, Lindberg MR, Kennett MJ, Xiong N, Wang Y. PAD4 is essential for antibacterial innate immunity mediated by neutrophil extracellular traps. J Exp Med 2010; 207: 1853-1862.

43. Behnen M, Leschczyk C, Moller S, Batel T, Klinger M, Solbach W et al. Immobilized immune complexes induce neutrophil extracellular trap release by human neutrophil granulocytes via FcgammaRIIIB and Mac-1. J Immunol 2014; 193: 1954-1965.

44. Lood C, Blanco LP, Purmalek MM, Carmona-Rivera C, De Ravin SS, Smith CK et al. Neutrophil extracellular traps enriched in oxidized mitochondrial DNA are interferogenic and contribute to lupus-like disease. Nat Med 2016; 22: 146-153.

45. Falk RJ, Terrell RS, Charles LA, Jennette JC. Anti-neutrophil cytoplasmic autoantibodies induce neutrophils to degranulate and produce oxygen radicals in vitro. Proc Natl Acad Sci USA 1990; 87: 4115-4119.

46. Primo VC, Marusic S, Franklin CC, Goldmann WH, Achaval CG, Smith RN et al. Anti-PR3 immune responses induce segmental and necrotizing glomerulonephritis. Clin Exp Immunol 2010; 159: 327-337.

47. Pradhan VD, Badakere SS, Bichile LS, Almeida AF. Anti-neutrophil cytoplasmic antibodies (ANCA) in systemic lupus erythematosus: prevalence, clinical associations and correlation with other autoantibodies. J Assoc Physicians India 2004; 52: 533-537.
48. Sakaguchi Y, Uehata T, Kawabata H, Niihata K, Shimomura A, Suzuki A et al. An autopsyproven case of myeloperoxidase-antineutrophil cytoplasmic antibody- positive polyarteritis nodosa with acute renal failure and alveolar hemorrhage. Clin Exp Nephrol 2011; 15: 281-284.

49. Ben Ameur S, Niaudet P, Baudouin V, Le Bourgeois M, Houdouin V, Delacourt C et al. Lung manifestations in MPO-ANCA associated vasculitides in children. Pediatr Pulmonol 2014; 49: 285-290.

50. Sai J, Raman D, Liu Y, Wikswo J, Richmond A. Parallel phosphatidylinositol 3-kinase (PI3K)dependent and Src-dependent pathways lead to CXCL8-mediated Rac2 activation and chemotaxis. J Biol Chem 2008; 283: 26538-26547.

51. Lim MB, Kuiper JW, Katchky A, Goldberg H, Glogauer M. Rac2 is required for the formation of neutrophil extracellular traps. J Leukoc Biol 2011; 90: 771-776.

52. Hochberg MC. Updating the American College of Rheumatology revised criteria for the classification of systemic lupus erythematosus. Arthritis Rheum 1997; 40: 1725.

53. Odobasic D, Gan PY, Summers SA, Semple TJ, Muljadi RC, Iwakura Y et al. Interleukin$17 \mathrm{~A}$ promotes early but attenuates established disease in crescentic glomerulonephritis in mice. Am J Pathol 2011; 179: 1188-1198.

54. Zhao W, Thacker SG, Hodgin JB, Zhang H, Wang JH, Park JL et al. The peroxisome proliferator-activated receptor gamma agonist pioglitazone improves cardiometabolic risk and renal inflammation in murine lupus. J Immunol 2009; 183: 2729-2740.

(c) (i) (2) This work is licensed under a Creative Commons Attribution-NonCommercial-ShareAlike 4.0 International License. The images or other third party material in this article are included in the article's Creative Commons license, unless indicated otherwise in the credit line; if the material is not included under the Creative Commons license, users will need to obtain permission from the license holder to reproduce the material. To view a copy of this license, visit http://creativecommons.org/licenses/by-nc-sa/4.0/

(C) The Author(s) 2017

Supplementary Information accompanies this paper on Cell Death and Differentiation website (http://www.nature.com/cdd) 